\title{
No relevant differences in conditioned pain modulation effects between parallel and sequential test design. A cross-sectional observational study
}

\author{
Roland R Reezigt ${ }^{\text {Corresp., } 1,2}{ }^{\text {, Sjoerd C. Kielstra }}{ }^{1}$, Michel W. Coppieters ${ }^{1,3}$, Gwendolyne G.M. Scholten-Peeters ${ }^{\text {Corresp. } 1}$ \\ ${ }^{1}$ Department of Human Movement Sciences, Faculty of Behavioural and Movement Sciences, Vrije Universiteit Amsterdam, Amsterdam, Netherlands \\ 2 Academy of Health, Department of Physiotherapy, Hanze University of Applied Sciences, Groningen, Netherlands \\ 3 Menzies Health Institute Queensland, Griffith University, Brisbane and Gold Coast, Australia \\ Corresponding Authors: Roland R Reezigt, Gwendolyne G.M. Scholten-Peeters \\ Email address: r.r.reezigt@vu.nl, g.g.m.scholten-peeters@vu.nl
}

Background. Conditioned pain modulation (CPM) is measured by comparing pain induced by a test stimulus with pain induced by the same test stimulus, either during (parallel design) or after (sequential design) the conditioning stimulus. Whether design, conditioning stimulus intensity and test stimulus selection affect CPM remains unclear.

Methods. CPM effects were evaluated in healthy participants $(N=89)$ at the neck, forearm and lower leg using the cold pressor test as the conditioning stimulus. In three separate experiments, we compared the impact of (1) design (sequential versus parallel), (2) conditioning stimulus intensity (VAS 40/100 versus VAS 60/100), and (3) test stimulus selection (single versus dual, i.e., mechanical and thermal). Statistical analyses of the main effect of design (adjusted for order) and experiment were conducted using linear mixed models with random intercepts.

Results. No significant differences were identified in absolute CPM data. In relative CPM data, a sequential design resulted in a slightly lower CPM effect compared to a parallel design, and only with a mechanical test stimulus at the neck $(-6.1 \% ; 95 \% \mathrm{Cl}:-10.1$ to -2.1$)$ and lower leg $(-5.9 \% ; 95 \% \mathrm{Cl}:-11.7$ to $-0.1)$ but not forearm $(-4.5 \% ; 95 \% \mathrm{Cl}:-9.0$ to 0.1$)$. Conditioning stimulus intensity and test stimulus selection did not influence the CPM effect nor the difference in CPM effects derived from parallel versus sequential designs.

Conclusions. Differences in CPM effects between protocols were minimal or absent. A parallel design may lead to a minimally higher relative CPM effect when using a mechanical test stimulus. The conditioning stimulus intensities assessed in this study and performing two test stimuli did not substantially influence the differences between designs nor the magnitude of the CPM effect. 


\section{No relevant differences in conditioned pain}

\section{2 modulation effects between parallel and sequential}

\section{3 test design. A cross-sectional observational study}

4

5

6

7

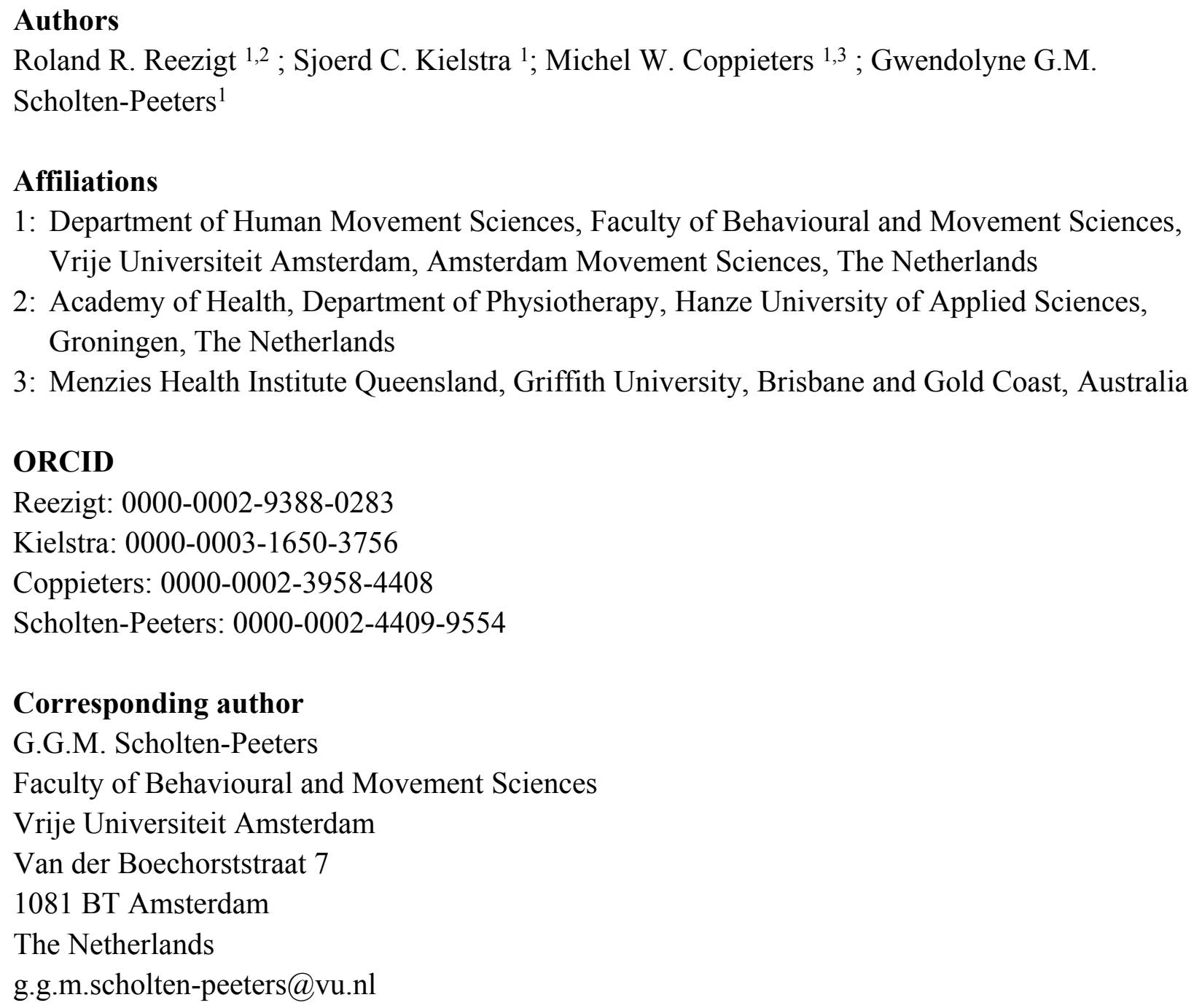




\section{Abstract}

33 Background. Conditioned pain modulation (CPM) is measured by comparing pain induced by a test stimulus with pain induced by the same test stimulus, either during (parallel design) or after (sequential design) the conditioning stimulus. Whether design, conditioning stimulus intensity and test stimulus selection affect CPM remains unclear.

Methods. CPM effects were evaluated in healthy participants $(N=89)$ at the neck, forearm and lower leg using the cold pressor test as the conditioning stimulus. In three separate experiments, we compared the impact of (1) design (sequential versus parallel), (2) conditioning stimulus intensity (VAS 40/100 versus VAS 60/100), and (3) test stimulus selection (single versus dual, i.e., mechanical and thermal). Statistical analyses of the main effect of design (adjusted for order) and experiment were conducted using linear mixed models with random intercepts.

Results. No significant differences were identified in absolute CPM data. In relative CPM data, a sequential design resulted in a slightly lower CPM effect compared to a parallel design, and only with a mechanical test stimulus at the neck (-6.1\%; 95\%CI: -10.1 to -2.1$)$ and lower leg $(-5.9 \%$; 95\%CI: -11.7 to -0.1$)$ but not forearm $(-4.5 \%$; $95 \% \mathrm{CI}:-9.0$ to 0.1$)$. Conditioning stimulus

47 intensity and test stimulus selection did not influence the CPM effect nor the difference in CPM effects derived from parallel versus sequential designs.

Conclusions. Differences in CPM effects between protocols were minimal or absent. A parallel design may lead to a minimally higher relative CPM effect when using a mechanical test

51 stimulus. The conditioning stimulus intensities assessed in this study and performing two test 52 stimuli did not substantially influence the differences between designs nor the magnitude of the 53 CPM effect. 


\section{Introduction}

55

Conditioned pain modulation (CPM) is of increasing interest in studies assessing central pain mechanisms [10]. CPM is a paradigm that tests the central pain inhibiting mechanism in response to a second nociceptive stimulus $[58,83]$. Patients with diverse pathologies, such as fibromyalgia [54], chronic low back pain [59], migraine [81], carpal tunnel syndrome [71], Achilles tendinopathy [75], and many chronic pain conditions [39] show a diminished CPM effect compared to healthy participants. This diminished effect, associated with less endogenous analgesia, may underlie persistent pain $[11,39,73,80]$.

For CPM quantification, pain induced by a test stimulus is compared to pain induced by the same test stimulus but applied during or immediately after a painful conditioning stimulus [52]. Different modalities of test stimuli and conditioning stimuli are used to measure CPM [31]. Commonly used test stimuli are mechanical (e.g., pressure pain thresholds and cuff pressure algometry $[33,69])$, and thermal stimuli (e.g., heat $[15,28])$. The cold pressor test $[23,66]$ and ischaemic pressure $[20,70]$ are frequently used conditioning stimuli. Further, after applying a first test stimulus (the baseline), the second test stimulus can be applied simultaneously with the conditioning stimulus (parallel design) $[26,40]$ or following the conditioning stimulus (sequential design) $[14,22]$. Even though many different CPM protocols are currently in use, it is unclear whether these different protocols have a substantial influence on the magnitude of the CPM effect and may partly explain discrepancies between CPM findings reported in the literature. These discrepancies in the CPM effect are observed in healthy participants (e.g., the effect may be anti-nociceptive, pro-nociceptive, or there may be no effect $[34,36,43,69])$. Furthermore, the size of the CPM effect may be inconsistent (e.g., elite athletes may show a smaller, similar or 
77 larger CPM effect compared to healthy participants [43], and similar inconsistencies are

78 observed within patient populations $[39,55,82])$.

Additionally, practical recommendations to measure the CPM effect have been formulated [84]. These recommendations suggest using both mechanical and thermal test stimuli within a protocol for comparability, and a sequential design to limit distraction bias while delivering the conditioning stimulus. Interestingly, the CPM effect duration after termination of the conditioning stimulus seems to be relatively short, varying from 0 to 10 minutes $[30,38,76]$. Local, regional and remote locations are often selected in clinical studies to differentiate between primary, secondary or widespread hyperalgesia or gain of nerve fibre function $[9,49,51,55,77]$. Therefore, the application of test stimuli on multiple locations takes time, which may justify using a parallel design where the duration of the conditioning stimulus can be extended until the last test stimulus [58]. Little is known however about potential differences in the magnitude of the CPM effect between a sequential or parallel design. Indirect studies reported conflicting results, showing no differences between both designs [29], larger effects for either a parallel $[48,60]$ or sequential [12] design. Moreover, statistical comparisons between the two designs were often not performed $[12,48,60]$.

Additionally, even though a mild to moderate pain intensity of the conditioning stimulus is sufficient to induce a CPM effect [52], it is unclear whether the intensity affects the magnitude of the CPM effect differently in the designs when the duration of the conditioning stimulus is different $[61,70]$. Moreover, it is unknown whether the combination of both mechanical and thermal test stimuli, as the recommendations prescribe [84], may interfere with each other. One test may lead to skin hyperalgesia which can influence the measurements necessary for the CPM effect $[44,62]$. 

recommendations [84], but substantiated deviations could be made. Therefore, this study aimed

102

103

to 1) assess possible differences in the magnitude of the CPM effect between a sequential and parallel design, using designs for clinical usage according to the recommendations with both a mechanical and thermal stimulus, 2) to evaluate the influence of conditioning stimulus intensity and the use of dual test stimuli (compared to single test stimuli) on a) potential differences between designs and $b$ ) on the CPM effect itself.

\section{Methods}

\section{Study design and planning}

A cross-sectional, double-blind observational study with a mixed-design was performed.

The study was approved by the scientific and ethical review board of the Vrije Universiteit Amsterdam, The Netherlands (VCWE-2017-022R1). The study was conducted according to the Declaration of Helsinki (2013).

To address the study aims, three separate experiments with different participant groups were conducted. In each experiment, both a sequential and parallel design was performed (as repeated measurement), and the cold-pressor test was used as the conditioning stimulus. In Experiment 1, a conditioning stimulus intensity of VAS 40/100 was used, with both mechanical and thermal test stimuli; Experiment 2 was identical to Experiment 1, but the conditioning stimulus intensity was VAS 60/100; Experiment 3 was identical to Experiment 2, but with only a mechanical test stimulus. Table 1 and Figure 1B provides an overview of the experiments. 
[Insert Fig. 1 approximately here]

125

For the first aim, the difference in CPM effect between parallel and sequential designs

127

128

129

130

131

132

133

134

135

136

137

138

139

140

141

142

143

144

145

146

147

was compared across all three experiments. For the second aim, the impact of the conditioning

stimulus intensity on CPM was assessed by comparing Experiment 1 and Experiment 2. The

impact of using single or dual test stimuli on the CPM effect was evaluated by comparing

Experiment 2 and Experiment 3.

The procedures were comparable between the three experiments, consisting of

familiarisation, baseline measurements and a sequential and parallel CPM design (see Figure 1

for an overview of the procedures). Prior to familiarisation, demographic data were collected.

Due to the length of the total procedure, possibly resulting in attention bias, the

measurements in Experiment 1 were performed on two consecutive days (the sequential design

on one day, the parallel design on another day, in random order). Participants were familiarised

with the procedures on the first testing day. All measurements were performed at approximately

the same time of the day to account for the possible influence of circadian rhythm [30]. The

procedure of Experiment 2 was identical to Experiment 1. Experiment 3 was performed in a single day due to a shorter protocol, with a 20 -minutes rest period between the designs.

\section{Participants}

Participants were recruited from the Academy of Health from the Hanze University of Applied Sciences in Groningen, The Netherlands. Participants had to be healthy and painfree[7,16], to avoid influences of health conditions or disorders on pain modulation.

Additionally, they had to be naive to pain modulation mechanisms to avoid potential expectation bias [53]. Additionally, due to the potential influence of hand dominance on pain sensitivity [57], 
148 all participants had to be right-handed. Recruitment was matched in the experimental groups for 149 age and sex to enable comparison [19,24,37]. Exclusion criteria were: acute pain, a history of

150 chronic pain or chronic pain syndromes, such as fibromyalgia, migraine or irritable bowel

151 syndrome, use of analgesic or psychiatric medication, Reynaud syndrome, intolerance for cold,

152 cardiovascular, respiratory, systematic or neurologic disease, pregnancy or inability to lie down

153 in a prone position for the duration of the experiment. Furthermore, participants who scored

154 more than the cut-off value of 10 on the Generalised Anxiety and Depression-7 item scale

155 (GAD-7) were excluded [2,72]. All participants provided written informed consent prior to 156 participating.

Participants were asked to refrain from pain medication, caffeine-containing products

$[4,65]$, alcohol $[74]$ and nicotine $[3,17]$ at least 24 hours before and during the measurements to reduce their influence on pain modulation. Participants were kept naïve to the study objective and were asked not to talk about the study with other participants to avoid potential expectation bias [53]. Furthermore, participants could only participate in one of the three experiments.

\section{Conditioned pain modulation}

Mechanical and thermal test stimuli were applied over the right paraspinal muscles at the C5-C6 level, extensor carpi radialis muscle and tibialis anterior muscle as these are frequently used test locations for CPM [34,50,67]. The cold pressor test was used as the conditioning stimulus. The left hand (with spread fingers) was submerged in cold water, at an individualised temperature. Participants were positioned in a prone position on a treatment plinth in a temperature-controlled and quiet room. The right arm was placed above the head with the elbow flexed. The right knee was flexed and fixated at the ankle by the rater while testing the lower leg. 
171 The prone position was chosen for a better stability compared to a sitting position during the 172 neck and arm measurements.

174 Test stimuli

As mechanical test stimulus, pressure pain thresholds (PPT) were measured with a digital algometer (Type II, Somedic AB, Stockholm, Sweden) using a $1 \mathrm{~cm}^{2}$ probe with an application 177 rate of $50 \mathrm{kPa} / \mathrm{s}$. The participants pressed the hand-held switch when the feeling of pressure changed into painful pressure [63]. The PPTs were assessed three times at each test location with an inter-stimulus period of 20 seconds [79].

As thermal test stimulus, a tonic heat test stimulus was delivered, using a $25 \mathrm{~mm} \times 50 \mathrm{~mm}$ $\left(12.5 \mathrm{~cm}^{2}\right)$ computer-controlled thermode (MSA Thermal Stimulator, Somedic AB, Stockholm, Sweden). The automatic maximal cut-off temperature was set at $50^{\circ} \mathrm{C}$ to prevent possible skin damage [63]. The method of levels was used at each location to determine the required temperature of the tonic heat test stimulus corresponding with a baseline pain of VAS 40/100 [40]. First, a temperature of $43^{\circ} \mathrm{C}$ for 10 seconds was used and the pain intensity was rated on a VAS. Then, the temperature was adjusted if needed by either $0.5^{\circ} \mathrm{C}, 1.0^{\circ} \mathrm{C}$ or $2.0^{\circ} \mathrm{C}$ for the next exposure to achieve the required pain intensity of VAS 40/100. The inter-stimulus period between these exposures was set at 60 s $[34,61]$. If $50^{\circ} \mathrm{C}$ was reached before the pain threshold was reached, $50^{\circ} \mathrm{C}$ was used.

During the baseline and conditioned measurements, all three locations were tested three 191 times for 30 seconds using the temperature obtained, to increase robustness and similarity to the mechanical stimulus. During each measurement, participants were asked to rate the pain on a 
193 VAS scale after 10, 20 and 30 seconds [19]. The time between the test locations was at least 20

194 seconds [19].

195 Measurements at the various locations were performed in a fixed cyclic order[5], starting

196 with the neck and ending with the tibialis anterior muscle. The time between measurement

197 procedures with the mechanical and thermal stimuli during the baseline as well as during the

198 conditioned measurements was 10 minutes to clear residual effects [84]. Mechanical and thermal

199 test stimuli were applied in random order. The time between the baseline and conditioned

200 measurements was pragmatically set at 20 minutes [19]. In Experiment 3, only one baseline

201 measurement was taken. Reasons for this were: the short timeframe in Experiment 3 compared to

202 Experiment 1 and 2 ( $\sim 1$ hour versus $\sim 24$ hours), the high reproducibility of the test stimulus [1]

203 and prevention of burden for the participants.

204

205

\section{Conditioning stimulus}

206

The cold pressor test was used as conditioning stimulus. The intra-session reliability of

measuring CPM with a cold pressure test is good to excellent $(\mathrm{ICC}=0.61-0.80)[31]$. A

calibrated, refrigerated circulating water bath with a capacity of 28 litres was used (Polyscience,

Illinois, USA). Since the perception of cold is individually different [21], the water temperatures

for the cold pressor test were tailored for the individual [55,69], such that VAS 40/100 was

reached in Experiment 1 and VAS 60/100 in Experiment 2 and Experiment 3.

To determine the water temperature for each participant, the starting temperature was set

213 at either $13^{\circ} \mathrm{C}$ or $17^{\circ} \mathrm{C}$ (Experiment 1 with VAS $\left.40 / 100\right)$ and $11^{\circ} \mathrm{C}$ or $15^{\circ} \mathrm{C}($ Experiment 2 and

214 Experiment 3 with VAS 60/100), based on the first experience with the cold water during

215 familiarisation (see figure 1A). The temperature was decreased at a rate of $\sim 0.2^{\circ} \mathrm{C} / \mathrm{s}$ until the 
216 target VAS was reached. The participants were asked to rate their pain intensity on a VAS every

21720 seconds until the target pain was reached.

218

219 Sequential and parallel design

220 In the sequential design, the hand was taken out of the cold water after 60 seconds and

221 dried with a towel by the research assistant [56,64]. In the parallel design, the conditioning

222 stimulus started 60 seconds before applying the test stimulus, and the hand was kept in the water

223 until all measurements of the test stimulus were performed (Figure 1C).

224

225

Familiarisation

226

Prior to the measurements on the first testing day, the participants were familiarised with

227

228

229

230

231

232

233

234

235

236

237

238 approximately one minute or until they perceived too much discomfort. Next, both the

mechanical and thermal test stimuli were applied on the dorsal side of the right hand to reduce

anxiety for the test stimuli [63]. Subsequently, the participants were familiarised with the

pressure pain threshold test procedure, including how to use the hand-held switch. Pressure pain

thresholds were applied once on each test location. Finally, the participants were familiarised

with the heat test stimulus procedure and the temperature corresponding to a VAS 40/100 was

determined. Standardised instructions were given to all participants. The break time between the familiarisation and the baseline measurements was set pragmatically at 20 minutes, equal to the break time between baseline and conditioned measurements [19]. 

sequential and parallel design, and the order of the thermal and mechanical test stimulus. An independent person performed the randomisation. The mechanical and thermal test stimuli were

242 applied by two testers (RR and SK) independently of each other and a research assistant applied

243 the conditioning stimulus.

Both testers were blinded to the measurement results and the sequential or parallel design, by using a screen between the treatment plinth and the cold water bath. The research assistant pretended to dry the participant's hand after 60 s in the parallel design, behind the screen, to guarantee blinding of the testers.

\section{Sample size}

Sample size calculations were based on a power of $\beta=.80$, a significance level of $\alpha=.05$ and a Generalised Linear Models (GLM) Repeated Measurements approach [6,47], using G*Power 3.1.9.2 [13]. For the main effect between the designs, a medium effect size was chosen $\left(\eta_{p}{ }^{2}=.09\right)$ as the minimal effect size to be found in the experiments. Consequently, in a $2 \times 3$ mixed-method design of two within-subjects (sequential and parallel) and three between-subjects groups (Experiment 1, 2 and 3), a minimal sample size of 87 participants was needed with at least 29 participants per experiment. Considering an anticipated maximal drop-out rate of 10\%

257 [15,32], 96 participants were recruited, with 32 participants per experiment.

\section{Data Analysis}

Demographics

We used descriptive analyses to report demographic data. All data, including residuals for analyses of variance (ANOVA), were tested for the assumption of normality using histograms, 
263 boxplots, Q-Q plots and the Shapiro-Wilk test. For continuous data with a normal distribution,

264 the mean and standard deviation (SD) were reported. Otherwise, the median and interquartile

265 range (IQR) or percentages were provided. Differences in demographic and baseline data

266 between the three experiments were analysed using Chi-Square, ANOVA or the non-parametric

267 Kruskal-Wallis H-test. Statistical analyses were performed in SPSS version 25 (IBM, Armonk,

268 NY, USA). The significance level for all tests was set at $\alpha=.05$.

269

270

\section{Calculations}

271

The test stimuli results were calculated as the mean of three measurements [34]. For the mechanical test stimulus, outliers (defined as a $20 \%$ difference from the mean of the other two measurements) were removed, and a fourth measurement was taken [75]. For the heat test stimulus, the mean was calculated of the VAS after 10, 20 and 30 seconds. and absolute difference between the mean baseline test and the mean conditioned test were both calculated as the recommendations prescribe [84]. denominator, subtracted by $100 \%$, so the relative CPM effect shows the deviation from the individuals' baseline (percent change). Using this calculation, a result of $0 \%$ reflects no difference between the baseline and conditioned pressure pain thresholds, and a positive value

282 reflects the presence of an inhibitory or anti-nociceptive CPM effect. Since the heat test measurement is a patient-reported VAS rating that decreases in case of a CPM effect, we used the same calculation but multiplied it by -1 , so that the CPM effects can be interpreted the same way for both types of stimuli. 
Absolute differences were calculated by taking the difference of the mean conditioned

287

288

289

290

291

292

293

294

295

296

297

298

299

300

301

302

303

304

305

306

307

308

test and the baseline pain threshold, in concordance with the relative CPM effects, so that

positive numbers indicate an inhibiting or anti-nociceptive CPM effect for both relative and absolute data, and negative numbers a facilitatory or pro-nociceptive effect.

\section{Statistical analyses}

Linear mixed model analyses with a Maximum Likelihood (ML) method were used to analyse differences in the magnitude of CPM effect between the sequential and parallel design, with the participants as a random effect. Random slopes were added to the random intercepts in case of significant differences between the models based on -2 log-likelihood tests. Design (sequential or parallel) and experiment (1,2 and 3) were considered as fixed variables. For the heat test stimulus, only Experiment 1 and Experiment 2 were used. Assumptions of linearity and residual normality were checked before modelling.

First, main effects of design and experiments were calculated per location. Since the designs were randomised, the effect of design was adjusted for the effect of order of measurements. This adjustment separates the effect of which design was used (sequential versus parallel) versus which design was used first (first versus second, irrespective of the design), providing a clearer representation of the main effect of design itself. Additionally, local effect sizes (Cohen's f², adjusted for order) were calculated for the main effect of design [68].

Analyses for the effect of experiment were conducted by using dummy variables, with Experiment 2 as control, where the contrast with Experiment 1 reflects the difference in intensity, and the contrast with Experiment 3 reflects the difference in using single or dual test stimulus. 
Next, interaction effects between the design and the experiments were calculated,

310

311

312

313

314

315

316

317

318

319

320

321

322

323

324

325

326

327

328

329

330

331

whereas a significant interaction effect reflects significant differences between the sequential and parallel designs due to the experimental conditions. The resulting coefficients, $95 \%$ confidence intervals and significance for the main and interaction effects are presented. The significance level for all tests was set at $\alpha=.05$.

Further, estimated marginal means of the absolute and relative magnitude of the CPM effect, including the differences between the sequential and parallel design, are presented in $\mathrm{kPa}$, VAS (0-100) or percentage.

Additionally, to assess the robustness of the results, whether differences between parallel and sequential testing are related to the distribution of the CPM effect itself, a quantile regression analysis was performed [8]. Quantile regression analysis enables to analyse beyond the mean of the sample, exploring the whole distribution, including the tails (pro-nociceptive or antinociceptive), of the target variable (CPM effect). Regression coefficients for the main effect of design are calculated per location and experiment at every $10^{\text {th }}$ quantile (a total of $9,10^{\text {th }}$ to $90^{\text {th }}$ ).

\section{Results}

In total, 96 participants were recruited, of whom 91 met the selection criteria; 89

participants completed the study: 29 in Experiment 1, 31 in Experiment 2 and 29 in

Experiment 3 (Figure 2). Participants' baseline characteristics were similar between the three experiments. No significant differences in baseline measurements between the three experimental were found. Sociodemographic and baseline measurements are summarised in Table 2. 
Magnitude of the CPM effect

For the linear mixed model analyses, the addition of random slopes did not improve the coefficients for the design and order effect. Table 4 shows the regression coefficients for the experiment effect and Table 5 presents all estimated marginal means of the CPM and the differences between the designs.

Absolute CPM effect

The absolute data showed no significant main effect for design or experiments for either the mechanical or thermal test stimulus on the CPM effect (Table 3 and Table 4). No interaction (design $\mathrm{x}$ experiment) was found either, meaning that the experimental condition did not influence a possible difference between the sequential and parallel design (mechanical (PPT); cervical spine, $p=.626$, forearm, $p=.395$, lower leg, $p=.402$, and for thermal; cervical spine, $p$ $=.540$, forearm, $p=.901$, lower leg, $p=.647$ ). Figure 3 shows the estimated marginal means of the absolute CPM effects. 
Relative CPM effect-Mechanical test stimulus

Contrary to the absolute data, the relative data showed a significant main effect at the neck for design of $\beta=-6.1 \%(95 \% \mathrm{CI}:-10.1$ to $-2.1 ; p=.003)$, indicating that the sequential design had a lower CPM effect compared to the parallel design. There was no significant main effect of experiment, indicating that the difference between the experiments in conditioning stimulus intensity (VAS 40 vs $60 / 100), \beta=3.5 \%(95 \%$ CI: -2.9 to $9.8 ; p=.282)$, or use of dual (mechanical and thermal) versus single (mechanical) test stimuli, $\beta=0.4 \%$ (95\% CI: -6.0 to 6.7 , $p=.908)$, did not affect the magnitude of the CPM effect. There was no significant interaction effect between design and experiments, $p=.547$, indicating that the difference between designs was observed in all three experiments comparably. At the forearm, there was no significant main effect for design, $\beta=-4.5 \%$ (95\% CI: -9.0 to $0.1, p=.055)$, or experiment. Neither the intensity, $\beta=-3.9 \%(95 \% \mathrm{CI}:-10.2$ to $2.3, p=.216)$, nor the dual stimulus, $\beta=0.1 \%(95 \% \mathrm{CI}:-6.2$ to 6.3 , $p=.984)$, had a significant effect. No interaction effect was found between design and experiments, $p=.944$, either. At the lower leg, there was a significant main effect for design, $\beta$ $=-5.9 \%(95 \% \mathrm{CI}:-11.7$ to $-0.1, p=.046)$, indicating a lower $\mathrm{CPM}$ effect for the sequential design. No significant main effect of experiment was present, for the comparison of using a lower intensity, $\beta=5.3 \%$ (95\% CI: -2.6 to $13.3, p=.185)$, or use of a single stimulus, $\beta=5.2 \%$ (95\% CI: $-2,8$ to $13.1,2.8, p=.197)$. There was no interaction effect (design $\mathrm{x}$ experiment), $p=$ .351 .

\section{Relative CPM effect - Thermal test stimulus}

For the heat test stimulus, there was no significant main effect for design at any test location; neck: $\beta=-5.1 \%(95 \% \mathrm{CI}:-13.4$ to $3.1, p=.214)$; forearm: $\beta=-1.7 \%(95 \% \mathrm{CI}$ : -9.8 to 
$3786.3, p=.670)$; lower leg, $\beta=-1.9 \%(95 \%$ CI: -8.6 to $4.9, p=.586)$. Main effects of the

379 experiments were not significant, indicating that a lower intensity of the conditioning stimulus

380 did not yield a significantly different CPM effect, at the neck, $\beta=4,6 \%$ (95\% CI: $-3,9$ to $13.2, p$

$381=.284)$, forearm, $\beta=3.5 \%(95 \% \mathrm{CI}:-4.6$ to $11.6, p=.392)$, or lower leg, $\beta=-0.1 \%(95 \% \mathrm{CI}$ : -

38210.1 to $10.0, p=.987)$. There were no significant interaction effects (design $\mathrm{x}$ experiment)

383 observed at the neck, $p=.546$, forearm, $p=.551$, or lower leg, $p=.552$. Figure 4 shows the

384 results of the relative CPM effects.

385

[Insert Fig. 4 approximately here]

[Insert Table 5 approximately here]

388

389

390

391

392

393

394

395

396

397

398

399

The effect of order

Main effects of design were adjusted for order effects, since the sequence in which the designs were tested was randomised. The order effect showed that the design which was performed first, irrespective of which design it was, resulted in a greater CPM effect (23 to 36 $\mathrm{kPa}$ for mechanical stimuli, $5 \mathrm{~mm} \operatorname{VAS}(0-100 \mathrm{~mm})$ for thermal testing). This finding was more apparent in the absolute data compared to the relative data (see Table 3).

Differences in effect of design between higher and lower levels of CPM Quantile regression analyses $\left(10^{\text {th }}\right.$ till $90^{\text {th }}$ quartile $)$ revealed no significant different regression coefficients compared to the main analyses, indicating that in pro-nociceptive up to anti-nociceptive CPM effects, no different main effects for design were found. Figure 5 shows the estimated regression coefficients (adjusted for order) and 95\% confidence intervals for the 
$40010^{\text {th }}$ till the $90^{\text {th }}$ quantiles including the estimated regression coefficient and $95 \%$ confidence

401 interval based on linear mixed model regression (main analysis) per outcome and location.

402

403

404

405

406

407

408

409

410

411

412

413

414

415

416

417

418

419

420

421

422

[Insert Fig. 5 approximately here]

\section{Discussion}

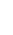

This study in healthy, young participants demonstrated that the magnitude of the CPM

effect is not significantly different between a parallel and sequential design. Only when

calculating the relative CPM effect, minimal, but no relevant higher effects were found for the

parallel design compared to the sequential design. All tested locations showed CPM effects of

comparable magnitudes. Additionally, experimental conditions, such as pain intensity (VAS 40

and 60/100) of the conditioning stimulus, or using a single (mechanical) versus dual (mechanical

and thermal) test stimuli, showed no significant influence on either the differences in the designs

or on the magnitude of the CPM effect itself.

The slightly higher relative CPM effects in the parallel design may partly be attributed to the substantially longer duration of the conditioning stimulus compared to the sequential design.

However, previous research showed conflicting results on the relation between duration of the conditioning stimulus and magnitude of the CPM effect[20,61,70]. One study found a higher CPM effect with a longer duration (6-12 minutes) of contact heat conditioning stimulus compared to a shorter duration (0-6 min), but only with a high intensity of the conditioning stimulus [61]. Contrary to other studies, no significant differences in CPM effect were found when using 10 s duration of cuff occlusion versus 60 s, and 20s versus 3 minutes [20,70].

Peer) reviewing PDF | (2021:05:61669:1:0:NEW 14 Sep 2021) 
424 or attention bias [84]. Attention, away from a pain-inducing stimulus, has a general analgesic

425 effect and might increase the measured CPM effect $[27,45,46]$. Additionally, focussing attention

426 on the conditioning stimulus gives a more potent inhibition compared to focussing attention on

427 the test stimulus $[35,43]$. It is therefore possible that attention bias has caused a slight

428 overestimation of the CPM-effect in our parallel design, when using a mechanical test stimulus.

429 Interestingly, we found no differences when using the thermal test stimulus, where we asked the

430 participants to rate the pain intensity every 10 seconds, possibly due to increased focus on the

431 test stimulus. Consequently, this could have decreased the influence of attention bias on the

432 conditioned stimulus leading to a more comparable construct as sequential testing. Further, since

433 the time in the cold water bath is considerably shorter using the sequential design (1 minute)

434 compared to the parallel design (5-7 minutes), this may lead to potentially less attention bias and 435 less burden to participants.

436 The effect of design was in most analyses smaller than the effect of order. The magnitude

437 of the CPM effect was slightly higher during the first measurement compared to the second

438 measurement, which could be explained by a habituation or salience effect due to the repetitive

439 nature of the study [25]. These observations are in line with a recent study which tested the CPM

440 effect two days after the first measurement in people with experimentally-induced low back pain

441 and found decreased CPM values on the second test occasion [42]. Additionally, the first

442 measurements influence the participant's expectation, which can influence the measurements on

443 the second day [18].

444 Differences in relative and absolute CPM effects can be explained by their different

445 calculations. Relative CPM effects use the unconditioned test (i.e., baseline PPT or heat 
446 threshold) as basis for the calculation. The outcome reflects the individual's pain inhibition,

447 since it is corrected for their baseline test result. For example, in people with low PPT or heat

448 baseline thresholds, small changes will lead to small absolute CPM effects, but can result in high

449 relative CPM effects as this change is compared to the individual's baseline. We observed this

450 effect on the group outcome (i.e., in the neck), where differences in designs were only observed

451 in the relative CPM effects and not in absolute CPM effects.

452 Our results showed no significant difference in CPM effect when different intensities of

453 the conditioning stimulus were used. We used VAS 40/100 (Experiment 1) and VAS 60/100

454 (Experiment 2 and 3) pain levels, in line with Nir et al. [52], who also found no differences in

455 magnitude of the CPM effect between these two intensities. Although no direct comparison can

456 be made, higher CPM effects were observed when using a much colder water bath $\left(\sim 3^{\circ} \mathrm{C}[69]\right.$

457 versus $\sim 12^{\circ} \mathrm{C}$ in Experiment 1 ; and $\sim 9^{\circ} \mathrm{C}$ in Experiment 2 and 3). This may be explained by the

458 experienced pain intensity using a $\sim 3^{\circ} \mathrm{C}$ water bath, which is probably outside the moderate-

459 intensity range used in our experiments. A high-intensity may have a different effect then

460 moderate-intensity and the differences between our intensities were not large enough to yield

461 results.

462

Although previous research found high anti-nociceptive CPM effects in healthy

463 participants $[39,69]$, an increasing number of studies find, in line with our study, low mean CPM

464 effects and large intra-individual variability $[33,36,55,78]$. One study with young, healthy adults

465 found a mean CPM magnitude of $\sim 0 \mathrm{kPa}$, similar to our results [33]. Their sample consisted of

466 two groups, young patients with low back pain and young, healthy controls. These authors

467 identified factors which were significantly associated with the absence of the CPM effect:

468 frequency and amount of alcohol consumption, and sleep disturbance and sleep latency. These 
469 factors, although not measured, can be anticipated to be also present in our participants as the

470 setting where the participants were recruited was comparable. These factors seem to influence

471 the CPM effect more than age, as previous research showed that the CPM effect should be higher

472 in younger people [24,69]. Future research is required to explore factors explaining the

473 variability of the magnitude of the CPM effect.

474 A repeated-measurement design with relatively long within-session rest periods could 475 have resulted in a loss of attention of our participants, especially for the last measurements of the 476 sessions. Therefore, we randomised the order of measurements (both test stimuli and both 477 designs) and statistically corrected for the order effect, but this could have resulted in lower 478 mean group CPM effects. The low mean CPM effects could potentially masque differences in 479 the designs.

Additional quantile regression analyses, however, showed no significantly different

481

482

483

484

485

486

487

488 489

490 results in estimated regression coefficient distribution, meaning that even in participants with higher (anti-nociceptive) or lower (pro-nociceptive) CPM effects, no significant differences between the sequential and parallel design were present.

Furthermore, we tried to reduce the influences of confounders by using a highly standardised protocol to control for variable conditions, including standardised communication with the participants and using strict selection criteria and balanced recruitment for age and sex. The strictness of the protocols used, may have reduced the generalisability of the measurements into clinical practice but was beneficial for the study's aim. 
This study compared a sequential and parallel design and found no relevant differences in 492 CPM effects between the two designs. No effect of conditioning stimulus intensity or the use of 493 dual test stimuli on a potential difference was found. Since the small differences in CPM effects 494 were only found in the relative data, it may be of importance to analyse and report both absolute 495 and relative data in future research, as reported in the recommendations for measuring CPM 496 effects.

497

498 


\section{Acknowledgements}

500

501 We would like to thank all participants and research assistants, in particular Caspar

502 Mylius, for their assistance with the project. 


\section{References}

504 [1] Arendt-Nielsen L, Waller R, Straker L, O'Sullivan P, Sterling M, Smith A, Arendt-Nielsen L, Waller R, Straker L, O'Sullivan P, Sterling M, Smith A. Reliability of pressure pain threshold testing (PPT)

506

507 in healthy pain free young adults. Scandinavian Journal of Pain 2015;9:28-29.

508 doi:10.1016/j.sjpain.2015.06.002.

508

Arntz A, Dreessen L, De Jong P. The influence of anxiety on pain: attentional and attributional mediators. Pain 1994;56:307-14.

510

Bagot KS, Wu R, Cavallo D, Krishnan-Sarin S. Assessment of pain in adolescents: Influence of gender, smoking status and tobacco abstinence. Addictive Behaviors 2017;67:79-85.

512

Baratloo A, Rouhipour A, Forouzanfar MM, Safari S, Amiri M, Negida A. The Role of Caffeine in Pain Management: A Brief Literature Review. Anesthesiology and Pain Medicine 2016;6.

[5] Bisset LM, Evans K, Tuttle N. Reliability of 2 protocols for assessing pressure pain threshold in

515 healthy young adults. Journal of Manipulative and Physiological Therapeutics 2015;38:282-287. doi:10.1016/j.jmpt.2015.03.001.

Chi YY, Glueck DH, Muller KE. Power and Sample Size for Fixed-Effects Inference in Reversible Linear Mixed Models. American Statistician 2019;73:350-359.

521

[8] Cook BL, Manning WG. Thinking beyond the mean: a practical guide for using quantile regression

[9] Courtney CA, Steffen AD, Fernández-de-las-Peñas C, Kim J, Chmell SJ. Joint Mobilization Enhances

Coghill RC, Yarnitsky D. Healthy and normal? The need for clear reporting and flexible criteria for Mechanisms of Conditioned Pain Modulation in Individuals With Osteoarthritis of the Knee. The Journal of orthopaedic and sports physical therapy 2016;46:1-30. Modulation to Placebo and Nocebo Effects in Experimental and Clinical Pain. International Review of Neurobiology. Academic Press Inc., 2018, Vol. 139. pp. 255-296.

11] Edwards RR. Individual differences in endogenous pain modulation as a risk factor for chronic pain. Neurology 2005;65:437-443.

[12] Enax-Krumova E, Plaga AC, Schmidt K, Özgül ÖS, Eitner LB, Tegenthoff M, Höffken O. Painful cutaneous electrical stimulation vs. Heat pain as test stimuli in conditioned pain modulation. Brain Sciences 2020;10:1-17.

[13] Faul F, Erdfelder E, Lang AG, Buchner A. G*Power 3: A flexible statistical power analysis program for the social, behavioral, and biomedical sciences. Behavior Research Methods 2007;39:175191.

[14] Flood A, Waddington G, Thompson K, Cathcart S. Increased conditioned pain modulation in athletes. Journal of Sports Sciences 2017;35. 
539

540

541

542

543

544

545

546

547

548

549

550

551

552

553

554

555

556

557

558

559

560

561

562

563

564

565

566

567

568

569

570

571

572

573

[15] Gehling J, Mainka T, Vollert J, Pogatzki-Zahn EM, Maier C, Enax-Krumova EK. Short-term testretest-reliability of conditioned pain modulation using the cold-heat-pain method in healthy subjects and its correlation to parameters of standardized quantitative sensory testing. BMC Neurology 2016;16:125.

[16] Gierthmühlen J, Enax-Krumova EK, Attal N, Bouhassira D, Cruccu G, Finnerup NB, Haanpää M, Hansson P, Jensen TS, Freynhagen R, Kennedy JD, Mainka T, Rice ASC, Segerdahl M, Sindrup SH, Serra J, Tölle T, Treede RD, Baron R, Maier C. Who is healthy? Aspects to consider when including healthy volunteers in QST-based studies - A consensus statement by the EUROPAIN and NEUROPAIN consortia. Pain 2015;156.

[17] Girdler SS, Maixner W, Naftel HA, Stewart PW, Moretz RL, Light KC. Cigarette smoking, stressinduced analgesia and pain perception in men and women. Pain 2005;114:372-385.

[18] Goffaux P, Redmond WJ, Rainville P, Marchand S. Descending analgesia - When the spine echoes what the brain expects. Pain 2007;130:137-143.

[19] Granot M, Weissman-Fogel I, Crispel Y, Pud D, Granovsky Y, Sprecher E, Yarnitsky D. Determinants of endogenous analgesia magnitude in a diffuse noxious inhibitory control (DNIC) paradigm: Do conditioning stimulus painfulness, gender and personality variables matter? Pain 2008;136:142-149. doi:10.1016/j.pain.2007.06.029.

[20] Graven-Nielsen T, Izumi M, Petersen KK, Arendt-Nielsen L. User-independent assessment of conditioning pain modulation by cuff pressure algometry. European journal of pain (London, England) 2017;21:552-561.

[21] Green BG, Akirav C. Threshold and rate-sensitivity of low-threshold thermal nociception. n.d.

[22] Grosen K, Vase L, Pilegaard HK, Pfeiffer-Jensen M, Drewes AM. Conditioned pain modulation and situational pain catastrophizing as preoperative predictors of pain following chest wall surgery: $\mathrm{A}$ prospective observational cohort study. PLOS ONE 2014;9.

[23] Grouper H, Eisenberg E, Pud D. The relationship between sensitivity to pain and conditioned pain modulation in healthy people. Neuroscience Letters 2019;708.

[24] Hackett J, Naugle KEKM, Naugle KEKM. The Decline of Endogenous Pain Modulation With Aging: A Meta-Analysis of Temporal Summation and Conditioned Pain Modulation. Journal of Pain 2020;21:514-528. doi:10.1016/j.jpain.2019.09.005.

[25] Hall G, Rodríguez G. Habituation and conditioning: Salience change in associative learning. Journal of Experimental Psychology: Animal Learning and Cognition 2017;43:48-61.

[26] Hoegh M, Petersen KK, Graven-Nielsen T. Effects of repeated conditioning pain modulation in healthy volunteers. European Journal of Pain (United Kingdom) 2018;22:1833-1843.

[27] Hoegh M, Seminowicz DA, Graven-Nielsen T. Delayed effects of attention on pain sensitivity and Conditioned Pain Modulation. European Journal of Pain 2019:ejp.1458. 
574 [28] Horn-Hofmann C, Kunz M, Madden M, Schnabel EL, Lautenbacher S. Interactive effects of

575

576

577

578

579

580

581

582

583

584

585

586

587

588

589

590

591

592

593

594

595

596

597

598

599

600

601

602

603

604

605

606

607

608 conditioned pain modulation and temporal summation of pain-the role of stimulus modality. Pain 2018;159:2641-2648.

[29] Ibancos-Losada MDR, Osuna-Pérez MC, Castellote-Caballero MY, Díaz-Fernández Á. Conditioned pain modulation effectiveness: An experimental study comparing test paradigms and analyzing potential predictors in a healthy population. Brain Sciences 2020;10:1-18.

[30] Imai Y, Petersen KK, Mørch CD, Arendt Nielsen L. Comparing test-retest reliability and magnitude of conditioned pain modulation using different combinations of test and conditioning stimuli. Somatosensory \& motor research 2016;0220:1-9.

[31] Kennedy DL, Kemp HI, Ridout D, Yarnitsky D, Rice ASC. Reliability of conditioned pain modulation: a systematic review. Pain 2016;157:2410-2419.

[32] Kjær Petersen K, Bjarke Vaegter H, Arendt-Nielsen L. An updated view on the reliability of different protocols for the assessment of conditioned pain modulation. Pain 2017;158.

[33] Klyne DM, Moseley GL, Sterling M, Barbe MF, Hodges PW. Individual Variation in Pain Sensitivity and Conditioned Pain Modulation in Acute Low Back Pain: Effect of Stimulus Type, Sleep, and Psychological and Lifestyle Factors. Journal of Pain 2018;19:942.e1-942.e18.

[34] Klyne DM, Schmid AB, Moseley GL, Sterling M, Hodges PW. Effect of types and anatomic arrangement of painful stimuli on conditioned pain modulation. Journal of Pain $2015 ; 16: 176-$ 185. doi:10.1016/j.jpain.2014.11.005.

[35] Ladouceur A, Tessier J, Provencher B, Rainville P, Piché M. Top-down attentional modulation of analgesia induced by heterotopic noxious counterstimulation. Pain 2012;153:1755-1762.

[36] Larsen JB, Madeleine P, Arendt-Nielsen L. Development of a new bed-side-test assessing conditioned pain modulation: A test-retest reliability study. Scandinavian Journal of Pain 2019;19:565-574.

[37] Leone C, Truini A. The CPM Effect: Functional Assessment of the Diffuse Noxious Inhibitory Control in Humans. Journal of Clinical Neurophysiology 2019;36:430-436.

[38] Lewis GN, Heales L, Rice DA, Rome K, McNair PJ. Reliability of the conditioned pain modulation paradigm to assess endogenous inhibitory pain pathways. Pain Research and Management 2012;17:98-102.

[39] Lewis GN, Rice DA, McNair PJ. Conditioned pain modulation in populations with chronic pain: A systematic review and meta-analysis. Journal of Pain 2012;13:936-944. doi:10.1016/j.jpain.2012.07.005.

[40] Lie MU, Matre D, Hansson P, Stubhaug A, Zwart J-A, Nilsen KB. A tonic heat test stimulus yields a larger and more reliable conditioned pain modulation effect compared to a phasic heat test stimulus. Pain reports 2017. 
609 [41] McDougall J, Jutzeler CR, Scott A, Crocker PRE, Kramer JLK. Conditioned pain modulation in elite

610

611

612

613

614

615

616

617

618

619

620

621

622

623

624

625

626

627

628

629

630

631

632

633

634

635

636

637

638

639

640

641

642

643

644

645 athletes: A systematic review and meta-analysis. Scandinavian Journal of Pain 2020;20:429-438. doi:10.1515/sjpain-2019-0153.

[42] McPhee M, Graven-Nielsen T. Alterations in Temporal Summation of Pain and Conditioned Pain Modulation Across an Episode of Experimental Exercise-Induced Low Back Pain. Journal of Pain 2018.

[43] Mertens MG, Hermans L, Geert Crombez M; , Goudman L, Calders P, Oosterwijck J van, Meeus M, Crombez G, Goudman L, Calders P, van Oosterwijck J, Meeus M. Comparison of five conditioned pain modulation paradigms and influencing personal factors in healthy adults. European Journal of Pain 2020. doi:10.1002/ejp.1665.

[44] Møiniche S, Dahl JB, Kehlet $\mathrm{H}$. Time course of primary and secondary hyperalgesia after heat injury to the skin. British Journal of Anaesthesia 1993;71:201-205.

[45] Moont R, Crispel Y, Lev R, Pud D, Yarnitsky D. Temporal changes in cortical activation during distraction from pain: A comparative LORETA study with conditioned pain modulation. Brain Research 2012;1435:105-117.

[46] Moont R, Pud D, Sprecher E, Sharvit G, Yarnitsky D. "Pain inhibits pain" mechanisms: Is pain modulation simply due to distraction? Pain 2010;150:113-120.

[47] Muller KE, Stewart PW. Linear Model Theory: Univariate, Multivariate, and Mixed Models. Wiley Blackwell, $2012 \mathrm{p}$.

[48] Nahman-Averbuch H, Granovsky Y, Coghill RC, Yarnitsky D, Sprecher E, Weissman-Fogel I. Waning of "conditioned pain modulation": A novel expression of subtle pronociception in migraine. Headache 2013;53:1104-1115.

[49] Nahman-Averbuch H, Thomas PL, Schneider VJ, Chamberlin LA, Peugh JL, Hershey AD, Powers SW, Coghill RC, King CD. Spatial aspects of pain modulation are not disrupted in adolescents with migraine. Headache: The Journal of Head and Face Pain 2020:head.14017.

[50] Neziri AY, Limacher A, Jüni P, Radanov BP, Andersen OK, Arendt-Nielsen L, Curatolo M. Ranking of tests for pain hypersensitivity according to their discriminative ability in chronic neck pain.

Regional anesthesia and pain medicine 2013;38:308-320.

[51] Ng TS, Pedler A, Vicenzino B, Sterling M. Less efficacious conditioned pain modulation and sensory hypersensitivity in chronic whiplash-Associated disorders in Singapore. Clinical Journal of Pain 2014;30.

[52] Nir RR, Granovsky Y, Yarnitsky D, Sprecher E, Granot M. A psychophysical study of endogenous analgesia: The role of the conditioning pain in the induction and magnitude of conditioned pain modulation. European Journal of Pain 2011;15:491-497.

[53] Nir RR, Yarnitsky D, Honigman L, Granot M. Cognitive manipulation targeted at decreasing the conditioning pain perception reduces the efficacy of conditioned pain modulation. Pain 2012;153:170-176. 
646 [54] O’Brien AT, Deitos A, Triñanes Pego Y, Fregni F, Carrillo-de-la-Peña MT. Defective Endogenous

647

648

649

650

651

652

653

654

655

656

657

658

659

660

661

662

663

664

665

666

667

668

669

670

671

672

673

674

675

676

677

678

679

680

Pain Modulation in Fibromyalgia: A Meta-Analysis of Temporal Summation and Conditioned Pain Modulation Paradigms. Journal of Pain 2018;19:819-836.

[55] Owens MA, Bulls HW, Trost Z, Terry SC, Gossett EW, Wesson-Sides KM, Goodin BR. An Examination of Pain Catastrophizing and Endogenous Pain Modulatory Processes in Adults with Chronic Low Back Pain. Pain Medicine n.d.;17:1452-1464. doi:10.1093/pm/pnv074.

[56] Perrotta A, Serrao M, Sandrini G, Burstein R, Sances G, Rossi P, Bartolo M, Pierelli F, Nappi G. Sensitisation of spinal cord pain processing in medication overuse headache involves supraspinal pain control. Cephalalgia 2010;30:272-284.

[57] Pud D, Golan Y, Pesta R. Hand dominancy-A feature affecting sensitivity to pain. Neuroscience Letters 2009;467:237-240.

[58] Pud D, Granovsky Y, Yarnitsky D. The methodology of experimentally induced diffuse noxious inhibitory control (DNIC)-like effect in humans. Pain 2009;144:16-19. doi:10.1016/j.pain.2009.02.015.

[59] Rabey M, Poon C, Wray J, Thamajaree C, East R, Slater H. Pro-nociceptive and anti-nociceptive effects of a conditioned pain modulation protocol in participants with chronic low back pain and healthy control subjects. Manual Therapy 2015;20.

[60] Ram KC, Eisenberg E, Haddad M, Pud D. Oral opioid use alters DNIC but not cold pain perception in patients with chronic pain - New perspective of opioid-induced hyperalgesia. Pain 2008;139:431-438. doi:10.1016/j.pain.2008.05.015.

[61] Razavi M, Hansson PTT, Johansson B, Leffler A-SS. The influence of intensity and duration of a painful conditioning stimulation on conditioned pain modulation in volunteers. European Journal of Pain 2014;18:853-861. doi:10.1002/j.1532-2149.2013.00435.x.

[62] Rgens TP, Sawatzki A, Henrich F, Magerl W, May A. An Improved Model of Heat-Induced Hyperalgesia-Repetitive Phasic Heat Pain Causing Primary Hyperalgesia to Heat and Secondary Hyperalgesia to Pinprick and Light Touch. PLoS ONE 2014;9:99507.

[63] Rolke R, Magerl W, Campbell KA, Schalber C, Caspari S, Birklein F, Treede RD. Quantitative sensory testing: A comprehensive protocol for clinical trials. European Journal of Pain 2006;10:77-88.

[64] Sandrini G, Rossi P, Milanov I, Serrao M, Cecchini AP, Nappi G. Abnormal modulatory influence of diffuse noxious inhibitory controls in migraine and chronic tension-type headache patients. Cephalalgia 2006;26:782-789.

[65] Sawynok J. Caffeine and pain. Pain 2011;152:726-729.

[66] Schliessbach J, Lütolf C, Streitberger K, Scaramozzino P, Arendt-Nielsen L, Curatolo M. Reference values of conditioned pain modulation. Scandinavian Journal of Pain 2019;19:279-286.

PeerJ reviewing PDF | (2021:05:61669:1:0:NEW 14 Sep 2021) 
681 [67] Scott D, Jull G, Sterling M. Widespread sensory hypersensitivity is a feature of chronic whiplash-

682

683

684

685

686

687

688

689

690

691

692

693

694

695

696

697

698

699

700

701

702

703

704

705

706

707

708

709

710

711

712

713

714

715

716 associated disorder but not chronic idiopathic neck pain. The Clinical journal of pain 2005;21:175-181.

[68] Selya AS, Rose JS, Dierker LC, Hedeker D, Mermelstein RJ. A practical guide to calculating Cohen's $\mathrm{f}$ 2, a measure of local effect size, from PROC MIXED. Frontiers in Psychology 2012;3.

[69] Skovbjerg S, Jørgensen T, Arendt-Nielsen L, Ebstrup JF, Carstensen T, Graven-Nielsen T. Conditioned Pain Modulation and Pressure Pain Sensitivity in the Adult Danish General Population: The DanFunD Study. Journal of Pain 2017;18:274-284. doi:10.1016/j.jpain.2016.10.022.

[70] Smith A, Pedler A. Conditioned pain modulation is affected by occlusion cuff conditioning stimulus intensity, but not duration. European Journal of Pain (United Kingdom) 2018;22:94-102.

[71] Soon B, Vicenzino B, Schmid AB, Coppieters MW. Facilitatory and inhibitory pain mechanisms are altered in patients with carpal tunnel syndrome. PLoS ONE 2017;12:1-11.

[72] Spitzer R, Kroenke K, Williams J, Lowe B. A brief measure for assessing generalized anxiety disorder. Arch Inern Med 2006;166:1092-7.

[73] Staud R. Abnormal endogenous pain modulation is a shared characteristic of many chronic pain conditions. Expert Review of Neurotherapeutics 2012;12:577-585.

[74] Thompson T, Oram C, Correll CU, Tsermentseli S, Stubbs B. Analgesic Effects of Alcohol: A Systematic Review and Meta-Analysis of Controlled Experimental Studies in Healthy Participants. Journal of Pain 2017;18:499-510.

[75] Tompra N, van Dieën JH, Coppieters MW. Central pain processing is altered in people with Achilles tendinopathy. British Journal of Sports Medicine 2015;50:1004-7. doi:10.1136/bjsports2015-095476.

[76] Vaegter HB, Handberg G, Graven-Nielsen T. Hypoalgesia After Exercise and the Cold Pressor Test is Reduced in Chronic Musculoskeletal Pain Patients With High Pain Sensitivity. The Clinical journal of pain 2016;32:58-69.

[77] Vaegter HB, Handberg G, Graven-Nielsen T. Similarities between exercise-induced hypoalgesia and conditioned pain modulation in humans. Pain 2014;155:158-167.

[78] Vaegter HB, Petersen KK, Mørch CD, Imai Y, Arendt-Nielsen L. Assessment of CPM reliability: Quantification of the within-subject reliability of 10 different protocols. Scandinavian Journal of Pain 2018;18:729-737.

[79] Visscher TLS, Lakerveld J, Olsen N, Küpers L, Ramalho S, Keaver L, Brei C, Bjune JI, Ezquerro S, Yumuk V. Perceived Health Status: Is Obesity Perceived as a Risk Factor and Disease? Obesity Facts 2017;10:52-60.

[80] van Wijk G, Veldhuijzen DS. Perspective on Diffuse Noxious Inhibitory Controls as a Model of Endogenous Pain Modulation in Clinical Pain Syndromes. Journal of Pain 2010;11:408-419. 
717 [81] Williams AE, Miller MM, Bartley EJ, McCabe KM, Kerr KL, Rhudy JL. Impairment of Inhibition of

718

719

720

721

722

723

724

725

726

727

728

729

730 Trigeminal Nociception via Conditioned Pain Modulation in Persons with Migraine Headaches. Pain medicine (Malden, Mass) 2019;20:1600-1610.

[82] Xie Y, Jun D, Thomas L, Coombes BK, Johnston V. Comparing Central Pain Processing in Individuals With Non-Traumatic Neck Pain and Healthy Individuals: A Systematic Review and Meta-Analysis. 2020. doi:10.1016/j.jpain.2020.02.007.

[83] Yarnitsky D, Arendt-Nielsen L, Bouhassira D, Edwards RR, Fillingim RB, Granot M, Hansson P, Lautenbacher S, Marchand S, Wilder-Smith O. Recommendations on terminology and practice of psychophysical DNIC testing. European Journal of Pain 2010;14:339.

[84] Yarnitsky D, Bouhassira D, Drewes AM, Fillingim RB, Granot M, Hansson P, Landau R, Marchand S, Matre D, Nilsen KB, Stubhaug A, Treede RD, Wilder-Smith OHG. Recommendations on practice of conditioned pain modulation (CPM) testing. European Journal of Pain (United Kingdom) 2015;19:805-806. 


\section{Table 1 (on next page)}

Overview of the three experiments

Overview of experiments with conditioning stimulus intensity and type of test stimulus used in the three experiments. VAS, Visual Analogue Scale 
Table 1 - Overview of the three experiments.

2

\begin{tabular}{|c|c|c|c|}
\hline & Experiment 1 & Experiment 2 & Experiment 3 \\
\hline Test Stimulus & $\begin{array}{l}\text { - Mechanical: } \\
\text { Pressure Pain Threshold } \\
\text { - Thermal: } \\
\text { Heat }\end{array}$ & $\begin{array}{l}\text { - Mechanical: } \\
\text { Pressure Pain } \\
\text { Threshold } \\
\text { - Thermal: } \\
\text { Heat }\end{array}$ & $\begin{array}{l}\text { - Mechanical: } \\
\text { Pressure Pain Threshold }\end{array}$ \\
\hline $\begin{array}{l}\text { Conditioning } \\
\text { stimulus Intensity } \\
\text { (Cold Pressor Test) }\end{array}$ & VAS 40/100 & VAS 60/100 & VAS 60/100 \\
\hline
\end{tabular}

Overview of experiments with conditioning stimulus intensity and type of test stimulus used in the three experiments. VAS, Visual Analogue Scale 


\section{Table 2 (on next page)}

Characteristics of the participants and baseline outcomes.

Data are presented as mean (SD) unless otherwise specified, ${ }^{\mathrm{a}}$ Median (IQR), ${ }^{\mathrm{b}}$ baseline measurements in Experiment3 are the same for both designs. ${ }^{c}$ No significant differences for sociodemographic data between the three experiments or between sequential versus parallel design BMI, body mass index; GAD-7, generalised anxiety disorder 7 items; PPT, Pressure Pain Threshold; kPa, kilo Pascal; CS, Conditioning Stimulus 
Table 2- Characteristics of the participants and baseline outcomes.

\begin{tabular}{|c|c|c|c|c|c|}
\hline & $\begin{array}{l}\text { Experiment } 1 \\
\text { Low conditioning } \\
\text { stimulus (VAS 40) / } \\
\text { dual test stimuli } \\
\end{array}$ & $\begin{array}{l}\text { Experiment } 2 \\
\text { High conditioning } \\
\text { stimulus (VAS 60) } \\
\text { / dual test stimuli } \\
\end{array}$ & $\begin{array}{l}\text { Experiment } 3 \\
\text { High conditioning } \\
\text { stimulus (VAS 60) / } \\
\text { single test stimulus } \\
\end{array}$ & Total & P-Value \\
\hline Demographics & $n=29$ & $n=31$ & $n=29$ & $N=89$ & \\
\hline Sex, male (\%) & $14(48)$ & $17(55)$ & $14(48)$ & $45(51)$ & $p=.896$ \\
\hline Age, years & $24(21-28)^{a}$ & $24(22-24)^{a}$ & $22(20-26)^{a}$ & $24(21-28)^{\mathrm{a}}$ & $p=.204$ \\
\hline Height, cm & $177(9)$ & $181(10)$ & $176(10)$ & $178(10)$ & $p=.377$ \\
\hline Weight, kg & 75 (12) & $74(12)$ & $71(68-79)^{\mathrm{a}}$ & $73(65-81)^{\mathrm{a}}$ & $p=.837$ \\
\hline BMI, $\mathrm{kg} / \mathrm{m}^{2}$ & $24.0(2.9)$ & $22.5(2.3)$ & $23.5(22.0-25.3)^{\mathrm{a}}$ & $\begin{array}{l}23.1(21.2- \\
25.2)^{\mathrm{a}}\end{array}$ & $p=.076$ \\
\hline Sports, hours/week & $5(2.5-6.5)^{\mathrm{a}}$ & $4(3-5)^{a}$ & $4.5(2.5-6.0)^{\mathrm{a}}$ & $4(3-6)^{\mathrm{a}}$ & $p=.328$ \\
\hline Anxiety, GAD-7 & $1(0-2.5)^{\mathrm{a}}$ & $2(0-3)^{\mathrm{a}}$ & $1(0-3)^{\mathrm{a}}$ & $1(0-3)^{\mathrm{a}}$ & $p=.377$ \\
\hline \multicolumn{6}{|l|}{$\begin{array}{l}\text { Baseline Pressure } \\
\text { Pain Threshold, kPa } \\
\text { Neck }\end{array}$} \\
\hline $\begin{array}{r}\text { Sequential } \\
\text { Parallel }\end{array}$ & $\begin{array}{l}418(143) \\
410(127)\end{array}$ & $\begin{array}{l}438(159) \\
476(183)\end{array}$ & $456(142)^{b}$ & $\begin{array}{l}438(148) \\
448(154)\end{array}$ & $\begin{array}{l}p=.632 \\
p=.242\end{array}$ \\
\hline $\begin{array}{r}\text { Sequential } \\
\text { Parallel }\end{array}$ & $\begin{array}{l}407(158) \\
395(118)\end{array}$ & $\begin{array}{l}398(138) \\
425(141)\end{array}$ & $456(140)^{\mathrm{b}}$ & $\begin{array}{l}420(146) \\
425(134)\end{array}$ & $\begin{array}{l}p=.262 \\
p=.232\end{array}$ \\
\hline $\begin{array}{r}\text { Sequential } \\
\text { Parallel }\end{array}$ & $\begin{array}{l}646(273) \\
577(496-690)^{\mathrm{a}}\end{array}$ & $\begin{array}{l}680(283) \\
705(276)\end{array}$ & $727(277)^{\mathrm{b}}$ & $\begin{array}{l}684(277) \\
688(280)\end{array}$ & $\begin{array}{l}p=.540 \\
p=.411\end{array}$ \\
\hline $\begin{array}{l}\text { Cold Pressor Test } \\
\text { Temperature CS }\left({ }^{\circ} \mathrm{C}\right)\end{array}$ & $11.9(11.7-12.0)^{\mathrm{a}}$ & $9.0(8.8-9.3)^{\mathrm{a}}$ & $9.9(9.4-10.7)$ & & \\
\hline
\end{tabular}


2 Data are presented as mean (SD) unless otherwise specified, ${ }^{a}$ Median (IQR), ${ }^{b}$ baseline measurements in Experiment 3 are the same for 3 both designs.

$4 \quad{ }^{\mathrm{c}}$ No significant differences for sociodemographic data between the three experiments or between sequential versus parallel design 5 BMI, body mass index; GAD-7, generalised anxiety disorder 7 items; PPT, Pressure Pain Threshold; kPa, kilo Pascal; CS,

6 Conditioning Stimulus 


\section{Table 3 (on next page)}

Effect of Design on magnitude of CPM effect, adjusted for order effect.

Significant effects are printed in bold. Test location at the cervical spine is paraspinal muscles over $\mathrm{C5/C6}$, at the forearm is extensor carpi radialis muscle and at the lower leg is the tibialis anterior muscle. A negative coefficient for design indicates the sequential design is lower compared to the parallel design. Order effect is based upon if the design was either first or second. A negative coefficient for order indicates that the first measurement is lower than the second measurement. ${ }^{\mathrm{a}} \mathrm{Absolute}$ data in $\mathrm{kPa} .{ }^{\mathrm{b}} \mathrm{Absolute}$ data in VAS $(0-100)$. 
Table 3 - Effect of Design on magnitude of CPM effect, adjusted for order effect.

\begin{tabular}{|c|c|c|c|c|c|c|c|c|c|c|}
\hline & \multirow[b]{3}{*}{ Location } & \multicolumn{5}{|c|}{ Absolute data } & \multicolumn{4}{|c|}{ Relative data (\%) } \\
\hline & & \multicolumn{3}{|c|}{ Design } & \multicolumn{2}{|c|}{ Order } & \multicolumn{3}{|c|}{ Design } & \multirow{2}{*}{$\begin{array}{r}\text { Order } \\
\beta, 95 \% \text { CI } \\
\end{array}$} \\
\hline & & $\beta, 95 \% \mathrm{CI}$ & P-Value & $\mathrm{f}^{2}$ & $\beta, 95 \% \mathrm{CI}$ & $\mathrm{P}$-value & $\beta, 95 \% \mathrm{CI}$ & P-Value & $\mathrm{f}^{2}$ & \\
\hline Mechanical & $\begin{array}{l}\text { Cervical } \\
\text { Spine }\end{array}$ & $-3[-21,15]$ & .723 & 0.00 & $24[6,43]$ & .009 & $-6.1[-10.1,-2.1]$ & .003 & 0.10 & $0.1[-3.9,4.1]$ \\
\hline Pressure Pain & Forearm & $5[-16,26]$ & .633 & 0.00 & $23[2,44]$ & .034 & $-4.5[-9.0,0.1]$ & .055 & 0.04 & $-0.7[-5.3,3.8]$ \\
\hline $\begin{array}{l}\text { Threshold } \\
(\text { PPT) })^{\mathrm{a}}\end{array}$ & Lower Leg & $-5[-40,30]$ & .780 & 0.00 & $46[11,81]$ & .010 & $-5.9[-11.7,-0.1]$ & .046 & 0.05 & $-0.3[-5.4,6.1]$ \\
\hline Thermal & $\begin{array}{l}\text { Cervical } \\
\text { Spine }\end{array}$ & $-1[-5,2]$ & .501 & 0.01 & $3[-1,6]$ & .120 & $-5.1[-13.4,3.1]$ & .214 & 0.03 & $6.3[-1.9,14.5]$ \\
\hline Heat Test & Forearm & $-1[-4,1]$ & .324 & 0.01 & $3[0,6]$ & .060 & $-1.7[-9.8,6.3]$ & .670 & 0.00 & $5.8[-2.2,13.9]$ \\
\hline 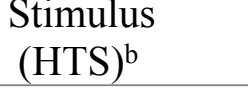 & Lower Leg & $1[-2,3]$ & .579 & 0.01 & $5[2,7]$ & $<.001$ & $-1.9[-8.6,4.9]$ & .586 & 0.00 & $9.2[2.4,15.9]$ \\
\hline
\end{tabular}

3 Significant effects are printed in bold. Test location at the cervical spine is paraspinal muscles over C5/C6, at the forearm is extensor carpi radialis muscle and at the lower leg is the tibialis anterior muscle. A negative coefficient for design indicates the sequential design is lower compared to the parallel design. Order effect is based upon if the design was either first or second. A negative coefficient for order indicates that the first measurement is lower than the second measurement. ${ }^{\mathrm{a}} \mathrm{Absolute}$ data in $\mathrm{kPa}$. ${ }^{\mathrm{b}} \mathrm{Absolute}$ data in VAS $(0-100)$. 


\section{Table 4 (on next page)}

Main effect of experiments on magnitude of CPM effect.

Significant effects are printed in bold. Test location at the cervical spine is paraspinal muscles over $\mathrm{C} 5 / \mathrm{C} 6$, at the forearm is extensor carpi radialis muscle and the lower leg is the tibialis anterior muscle. Experiment1; both test stimuli, conditioning stimulus VAS 40/100, Experiment2 (control); both test stimuli, conditioning stimulus VAS 60/100, Experiment3; only mechanical test stimulus, conditioning stimulus VAS 60/100. Coefficients shown in comparison with Experiment 2 as control, resulting in the coefficients of Experiment 1 reflecting the difference in the intensity of the conditioning stimulus and the coefficients of Experiment 3 reflecting the difference of single or dual test stimuli. Thermal testing was absent in Experiment 3. ${ }^{\mathrm{a}}$ Absolute data in $\mathrm{kPa} .{ }^{\mathrm{b}} \mathrm{Abs}$ blute data in VAS $(0-100)$. 
Table 4 - Main effect of experiments on magnitude of CPM effect.

\begin{tabular}{|c|c|c|c|c|c|c|c|c|}
\hline & \multirow[b]{3}{*}{ Location } & \multicolumn{4}{|c|}{ Absolute data } & \multicolumn{3}{|c|}{ Relative data (\%) } \\
\hline & & \multicolumn{2}{|c|}{ Experiment 1} & \multicolumn{2}{|c|}{ Experiment 3} & \multicolumn{2}{|c|}{ Experiment 1} & \multirow{2}{*}{$\begin{array}{l}\text { Experiment } 3 \\
\beta, 95 \% \mathrm{CI}\end{array}$} \\
\hline & & $\beta, 95 \% \mathrm{CI}$ & P-value & $\beta, 95 \% \mathrm{CI}$ & $\begin{array}{c}\text { P- } \\
\text { Value }\end{array}$ & $\beta, 95 \% \mathrm{CI}$ & P-Value & \\
\hline Mechanical & $\begin{array}{l}\text { Cervical } \\
\text { Spine }\end{array}$ & $17[-15,48]$ & .296 & $-21[-53,11]$ & .189 & $3.5[-2.9,9.8]$ & .282 & $0.4[-6.0,6.7]$ \\
\hline Pressure Pain & Forearm & $-19[-52,14]$ & .260 & $19[-52 ; 14]$ & .252 & $-3.9[-10.2,2.3]$ & .216 & $0.1[-6.2,6.3]$ \\
\hline $\begin{array}{l}\text { Threshold } \\
(\mathrm{PPT})^{\mathrm{a}}\end{array}$ & Lower Leg & $47[-14,108]$ & .132 & $-74[-135,-13]$ & .018 & $5.3[-2.6,13.3]$ & .185 & $5.2[-2.8,13.1]$ \\
\hline Thermal & $\begin{array}{l}\text { Cervical } \\
\text { Spine }\end{array}$ & $1[-2,5]$ & .476 & - & - & $4.6[-3.9,13.2]$ & .284 & - \\
\hline Heat Test & Forearm & $0[-3,3]$ & .773 & - & - & $3.5[-4.6,11.6]$ & .392 & - \\
\hline 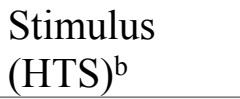 & Lower Leg & $-1[-4,1]$ & .321 & - & - & $-0.1[-10.1,10.0]$ & .987 & - \\
\hline
\end{tabular}

Significant effects are printed in bold. Test location at the cervical spine is paraspinal muscles over C5/C6, at the forearm is extensor carpi radialis muscle and at the lower leg is the tibialis anterior muscle. Experiment 1; both test stimuli, conditioning stimulus VAS 40/100, Experiment 2 (control); both test stimuli, conditioning stimulus VAS 60/100, Experiment 3; only mechanical test stimulus, conditioning stimulus VAS 60/100. Coefficients shown in comparison with Experiment 2 as control, resulting in the coefficients of Experiment 1 reflecting the difference in the intensity of the conditioning stimulus and the coefficients of Experiment 3 reflecting the difference of single or dual test stimuli. Thermal testing was absent in Experiment 3. ${ }^{\mathrm{a}} \mathrm{Absolute}$ data in $\mathrm{kPa}$. ${ }^{\mathrm{b}} \mathrm{Absolute}$ data in VAS (0 $-100)$. 


\section{Table 5 (on next page)}

Estimated marginal means of the of CPM effect (adjusted for order effect) in a sequential versus parallel design.

Estimated marginal means are adjusted for order effect. Significant differences are printed in bold. Relative data of $0.0 \%$ indicates that the baseline test and conditioned test were the same; no CPM effect is present. Positive values reflect an inhibitory effect for both absolute and relative data. Test location at the cervical spine is paraspinal muscles over $C 5 / C 6$, at the forearm is extensor carpi radialis muscle and at the lower leg is the tibialis anterior muscle.

CPM, Conditioned Pain Modulation; kPa, kilo Pascal; VAS, Visual Analogue Scale. ${ }^{a} p<.05$ 
Table 5 - Estimated marginal means of the of CPM effect (adjusted for order effect) in a sequential versus parallel design.

\begin{tabular}{|c|c|c|c|c|c|c|c|}
\hline \multicolumn{2}{|c|}{$\begin{array}{l}\text { Pressure Pain Threshold } \\
\text { (PPT) }\end{array}$} & \multicolumn{3}{|c|}{ Absolute CPM effect ( $\mathrm{kPa}, 95 \% \mathrm{CI})$} & \multicolumn{3}{|c|}{ Relative CPM effect $(\%, 95 \% \mathrm{CI})$} \\
\hline \multirow{5}{*}{$\begin{array}{l}\text { Cervical } \\
\text { spine }\end{array}$} & & Sequential & Parallel & Difference & Sequential & Parallel & Difference \\
\hline & Overall & $1[-15,17]$ & $4[-11,20]$ & $-3[-21,15]$ & $-2.4[-5.7,0.9]$ & $3.7[0.4,7.0]$ & $-6.1[-10.1,-2.1]^{a}$ \\
\hline & Experiment 1 & $2[-19,24]$ & $12[-9,33]$ & $-10[-37,17]$ & $0.0[-5.1,5.1]$ & $5.8[0.6,10.9]$ & $-5.8[-11.9,0.4]$ \\
\hline & Experiment 2 & $-10[-34,15]$ & $-9[-34,15]$ & $-0[-35,35]$ & $-3.1[-8.8,2.7]$ & $1.9[-3.9,7.7]$ & $-5.0[-13.1,3.2]$ \\
\hline & Experiment 3 & $11[-24,47]$ & $12[-24,47]$ & $-0[-31,30]$ & $-4.1[-10.3,2.2]$ & $3.6[-2.6,9.9]$ & $-7.7[-12.8,-2.6]^{a}$ \\
\hline \multirow[t]{4}{*}{ Forearm } & Overall & $12[-6,29]$ & $7[-11,24]$ & $5[-16,26]$ & $-1.2[-4.7,2.3]$ & $3.3[-0.2,6.7]$ & $-4.5[-9.1,0.2]$ \\
\hline & Experiment 1 & $-5[-30,19]$ & $-14[-39,10]$ & $9[-25,44]$ & $-3.8[-9.2,1.6]$ & $0.5[-4.9,5.9]$ & $-4.3[-11.9,3.3]$ \\
\hline & Experiment 2 & $16[-9,40]$ & $2[-22,27]$ & $13[-22,48]$ & $0.1[-6.2,6.0]$ & $4.7[-1.4,10.8]$ & $-4.8[-13.4,3.8]$ \\
\hline & Experiment 3 & $24[-15,64]$ & $32[-7,72]$ & $-8[-39,23]$ & $0.2[-6.3,6.6]$ & $4.5[-1.9,11.0]$ & $-4.4[-9.4,0.6]$ \\
\hline \multirow[t]{4}{*}{ Lower leg } & Overall & $42[11,73]$ & $47[16,78]$ & $-5[-40,30]$ & $4.2[-0.2,8.6]$ & $10.1[5.7,14.5]$ & $-5.9[-11.8,-0.0]^{a}$ \\
\hline & Experiment 1 & $53[6,99]$ & $52[5,98]$ & $1[-62,64]$ & $5.2[-2.6,13.0]$ & $13.0[5.1,20.8]$ & $-7.7[-18.5,3.0]$ \\
\hline & Experiment 2 & $10[-43,62]$ & $1[-52,53]$ & $9[-64,82]$ & $2.7[-5.8,11.3]$ & $4.8[-3.8,13.3]$ & $-2.0[-14.1,10.1]$ \\
\hline & Experiment 3 & $66[6,127]$ & $92[32,153]$ & $-26[-73,20]$ & $4.8[-1.3,10.8]$ & $13.1[7.0,19.2]$ & $-8.4[-13.0,-3.8]^{a}$ \\
\hline \multirow{2}{*}{\multicolumn{2}{|c|}{$\begin{array}{l}\text { Heat Test Stimulus } \\
\text { (HTS) }\end{array}$}} & \multicolumn{3}{|c|}{ Absolute CPM Effect (VAS 0-100, 95\% CI) } & \multicolumn{3}{|c|}{ Relative CPM Effect $(\%, 95 \%$ CI $)$} \\
\hline & & Sequential & Parallel & Difference & Sequential & Parallel & Difference \\
\hline Cervical & Overall & $3[0,6]$ & $4[2,7]$ & $-1[-5,2]$ & $5.2[-0.7,11.1]$ & $10.4[4.5,16.3]$ & $-5.1[-13.4,3.1]$ \\
\hline \multirow[t]{2}{*}{ Spine } & Experiment 1 & $4[1,8]$ & $4[1,8]$ & $-0[-5,5]$ & $9.0[1.0,17.0]$ & $11.4[3.4,19.4]$ & $-2.4[-13.7,8.9]$ \\
\hline & Experiment 2 & $2[-2,5]$ & $4[1,8]$ & $-2[-7,2]$ & $1.5[-7.0,10.1]$ & $9.6[1.0,18.2]$ & $-8.1[-18.7,2.5]$ \\
\hline \multirow[t]{3}{*}{ Forearm } & Overall & $2[-0,4]$ & $3[1,5]$ & $-1[-4,1]$ & $2.9[-2.8,8.6]$ & $4.7[-1.0,10.3]$ & $-1.7[-9.8,6.3]$ \\
\hline & Experiment 1 & $2[-1,5]$ & $3[1,6]$ & $-1[-5,3]$ & $3.6[-4.0,11.2]$ & $7.6[0.0,15.2]$ & $-4.1[-14.8,6.7]$ \\
\hline & Experiment 2 & $1[-1,4]$ & $3[1,6]$ & $-2[-5,2]$ & $2.2[-6.2,10.6]$ & $2.0[-6.4,10.4]$ & $-0.1[-10.6,10.9]$ \\
\hline Lower & Overall & $2[-2,4]$ & $3[1,5]$ & $-1[-4,1]$ & $2.8[-3.2,8.8]$ & $5.4[-0.1,11.4]$ & $-2.6[-9.3,4.1]$ \\
\hline
\end{tabular}




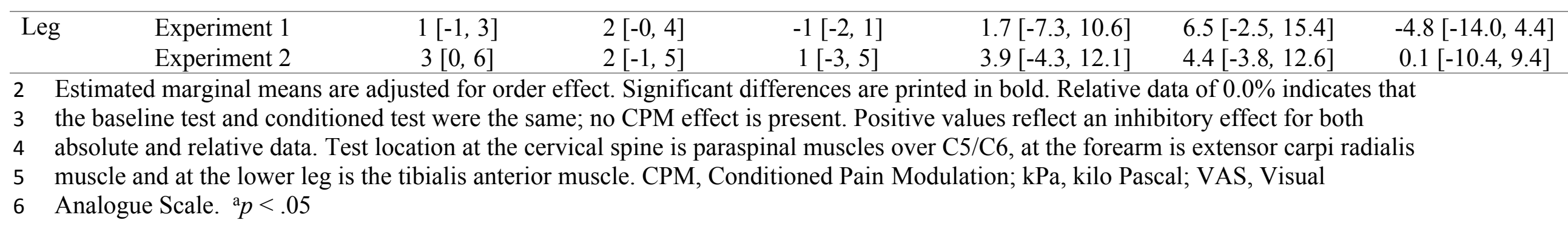




\section{Figure 1}

Overview of the study procedures, including the sequential and parallel design, for both mechanical and thermal test stimuli.

(A) Familiarisation with all stimuli (same for all experiments). (B) Baseline measures and conditioned measures with an overview of all three experiments (N.B. participants were enrolled in only one experiment, where both designs were performed). (C) Detail of the designs. In the sequential design, the conditioning stimulus was terminated after 60 seconds. The time between testing locations was 20 seconds. Arrows indicate measurement of VAS every 10 seconds per heat exposure of 30 seconds. Test locations; Neck: paraspinal muscles level C5/C6; Arm: extensor carpi radialis muscle; Leg: tibialis anterior muscle. 


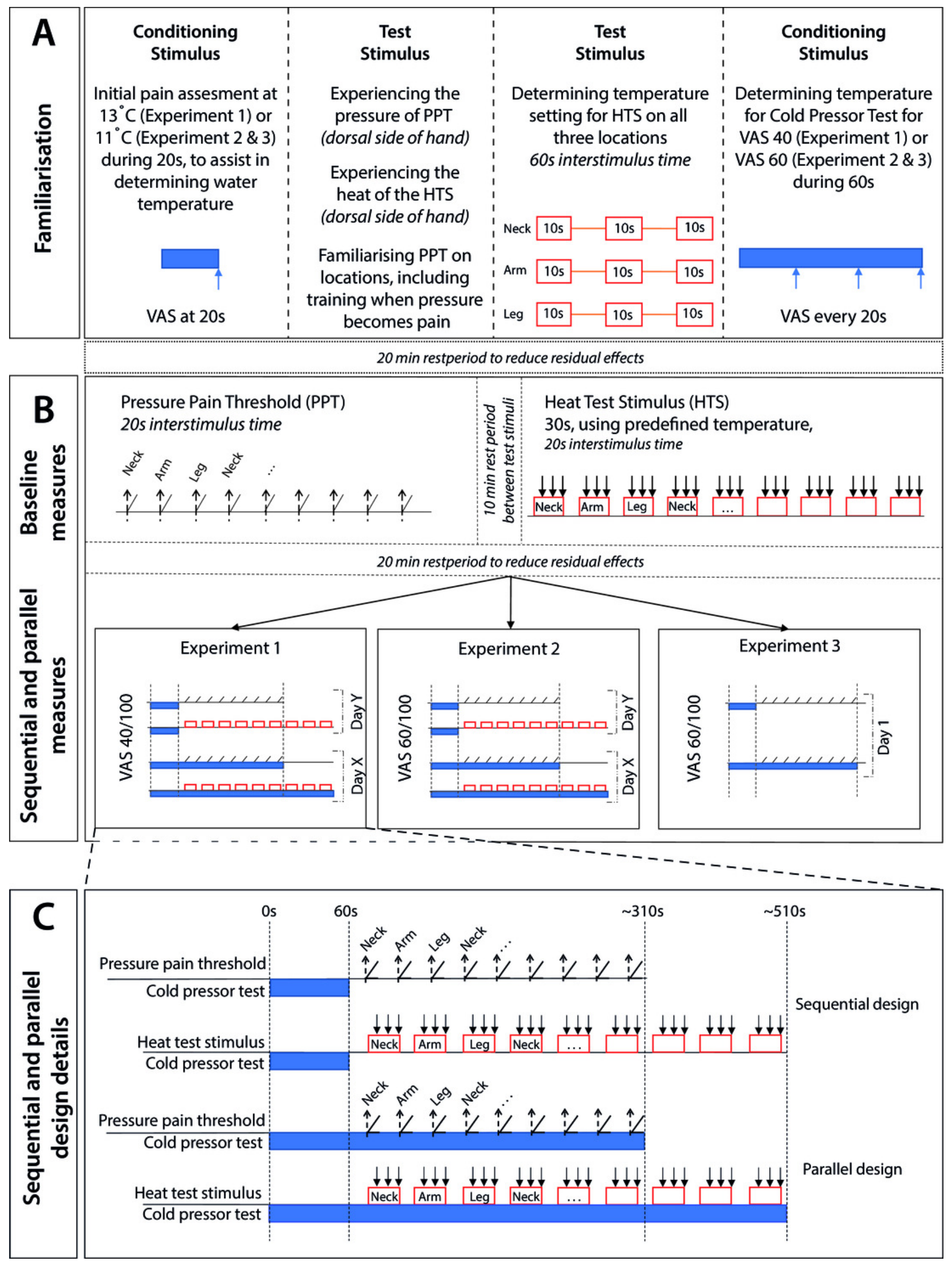


Figure 2

Flowchart of the study including all three experiments.

Flowchart showing the number of participants within each experiment and reasons for exclusion or lost on the second day. GAD-7, Generalised Anxiety Disorder 7 items

Experiment 1

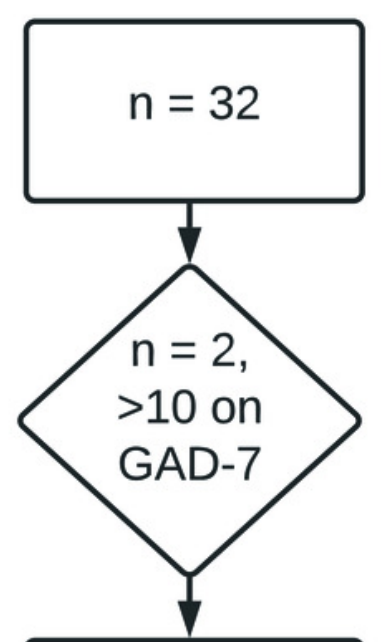

Measured on

Day 1

Not measured on

Day 2

Measured on

Day 1 \& Day 2
Experiment 2

Experiment 3

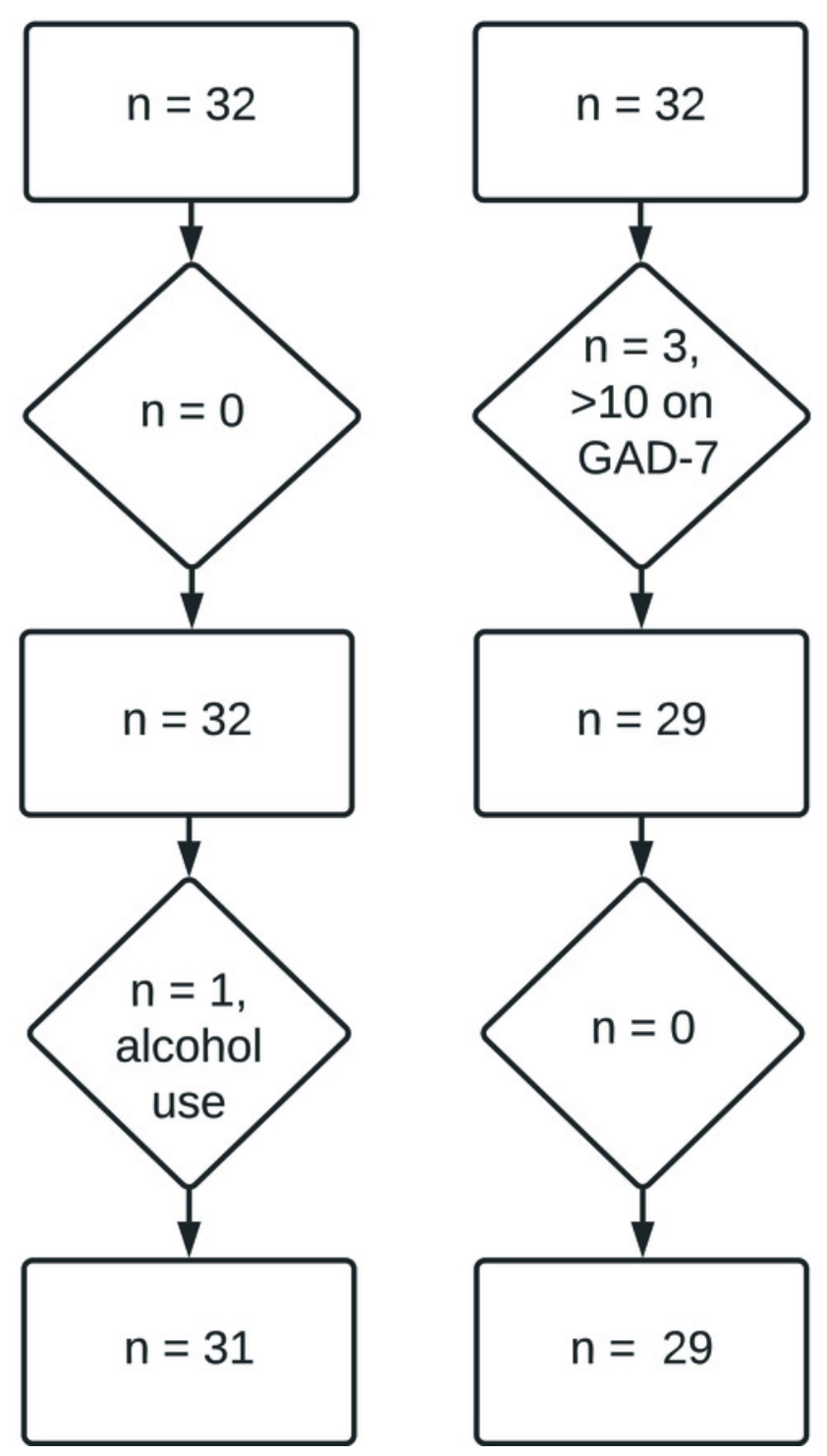


Figure 3

Absolute CPM effects.

Absolute CPM effect based on estimated marginal means with a mechanical and thermal test stimulus for the sequential (blue) and parallel (red) design per experiment, including 95\% confidence intervals, presented per location. Positive values reflect an inhibitory effect and negative values a facilitatory effect. 


\section{Absolute CPM effect}

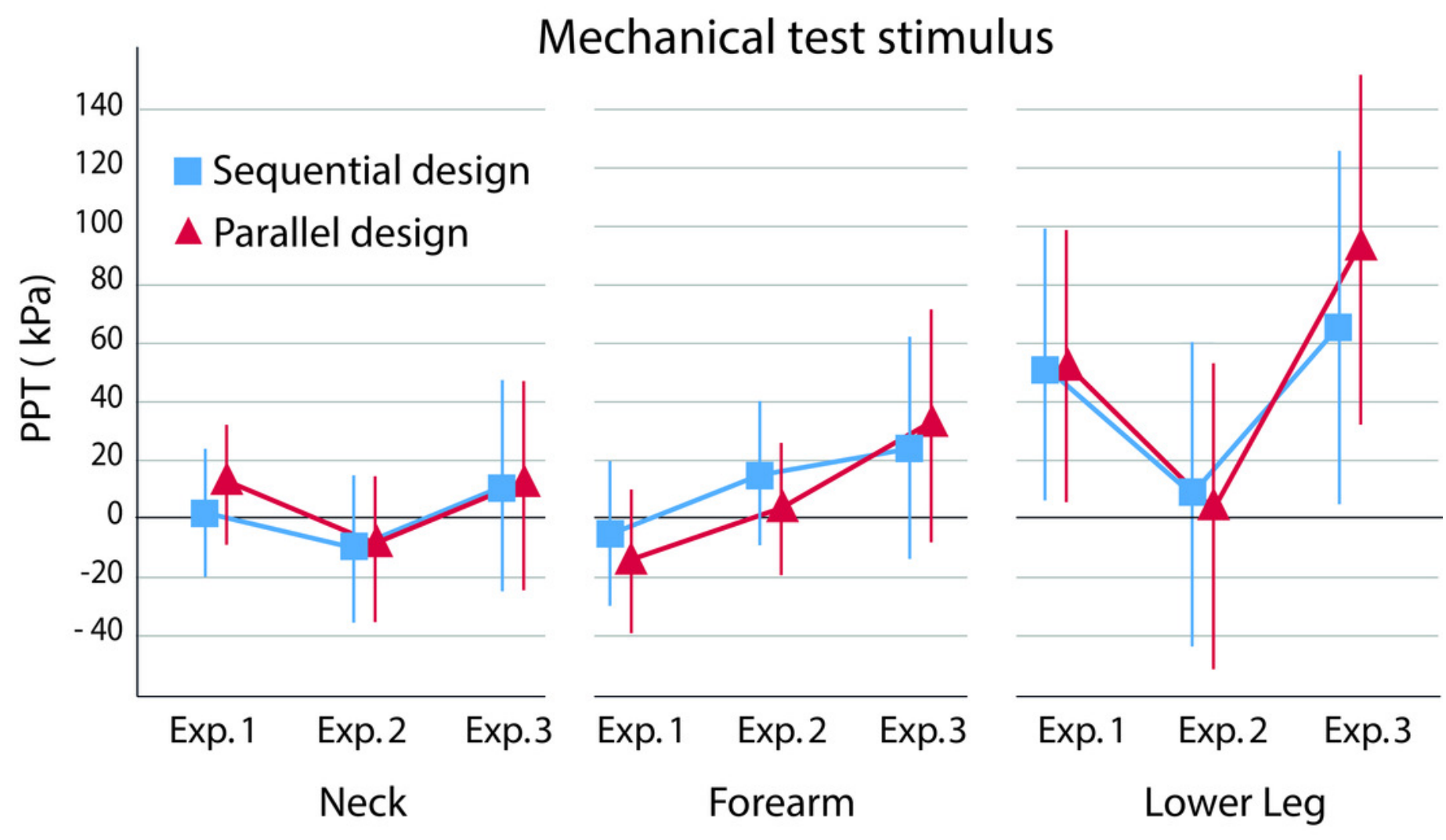

Thermal test stimulus
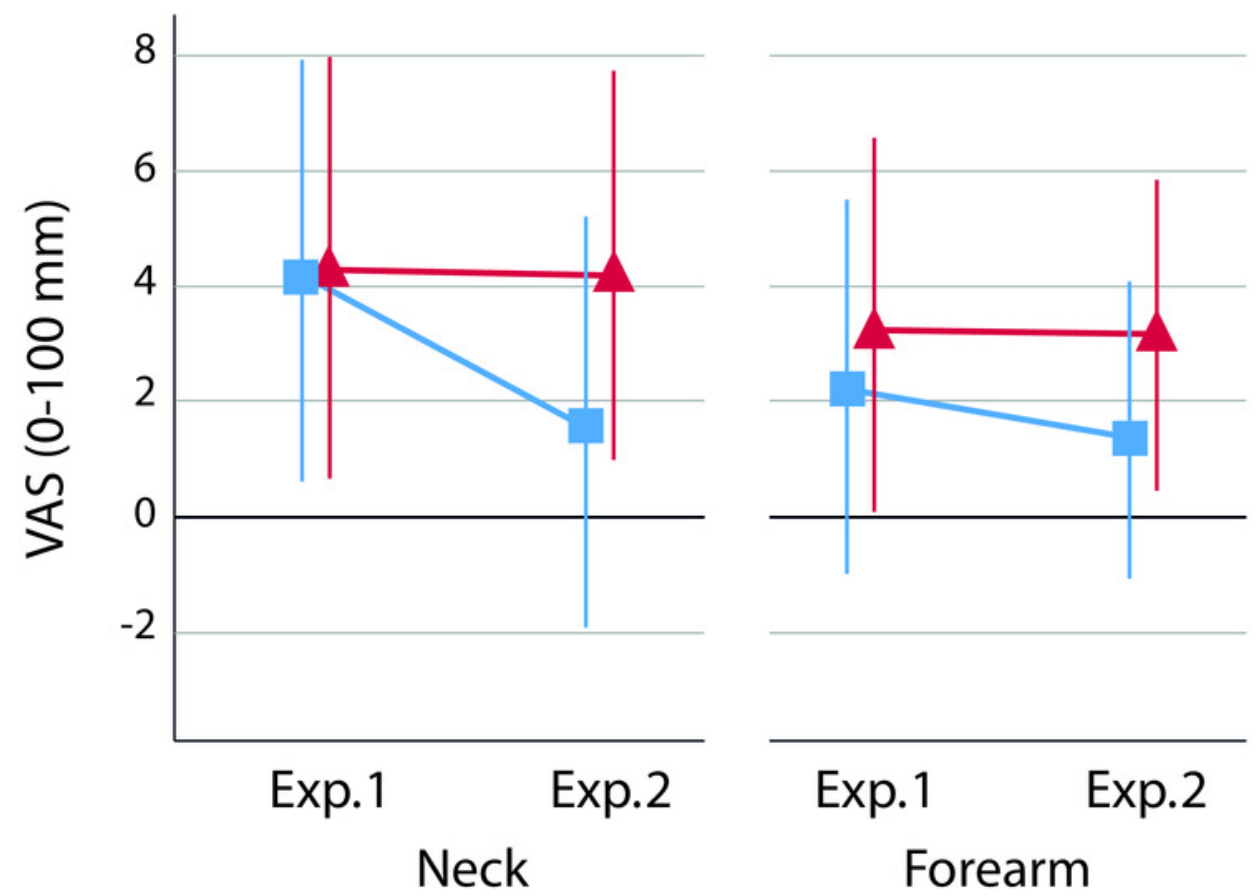

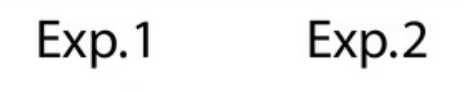

Forearm

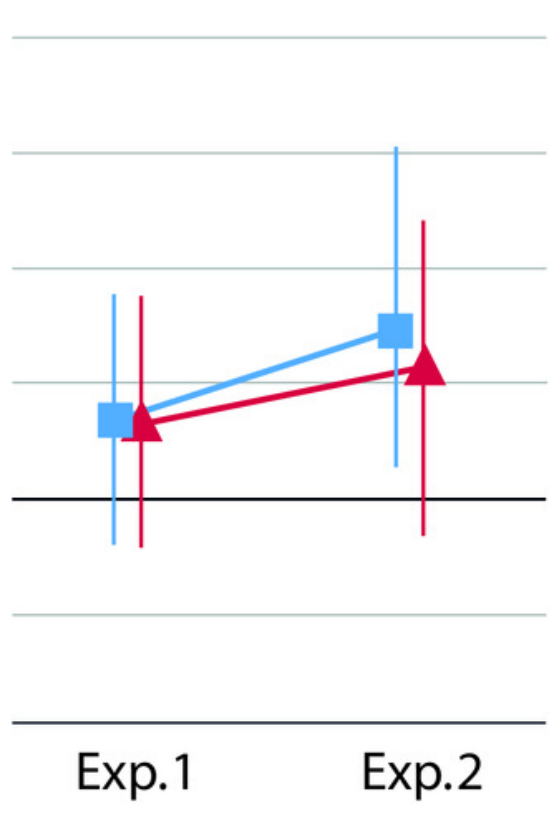

Lower Leg 
Figure 4

Relative CPM effects.

Relative CPM effect based on estimated marginal means with a mechanical and thermal test stimulus for the sequential (blue) and parallel (red) design per experiment, including 95\% confidence intervals, presented per location. Positive values reflect an inhibitory effect and negative values a facilitatory effect. 


\section{Relative CPM effect}

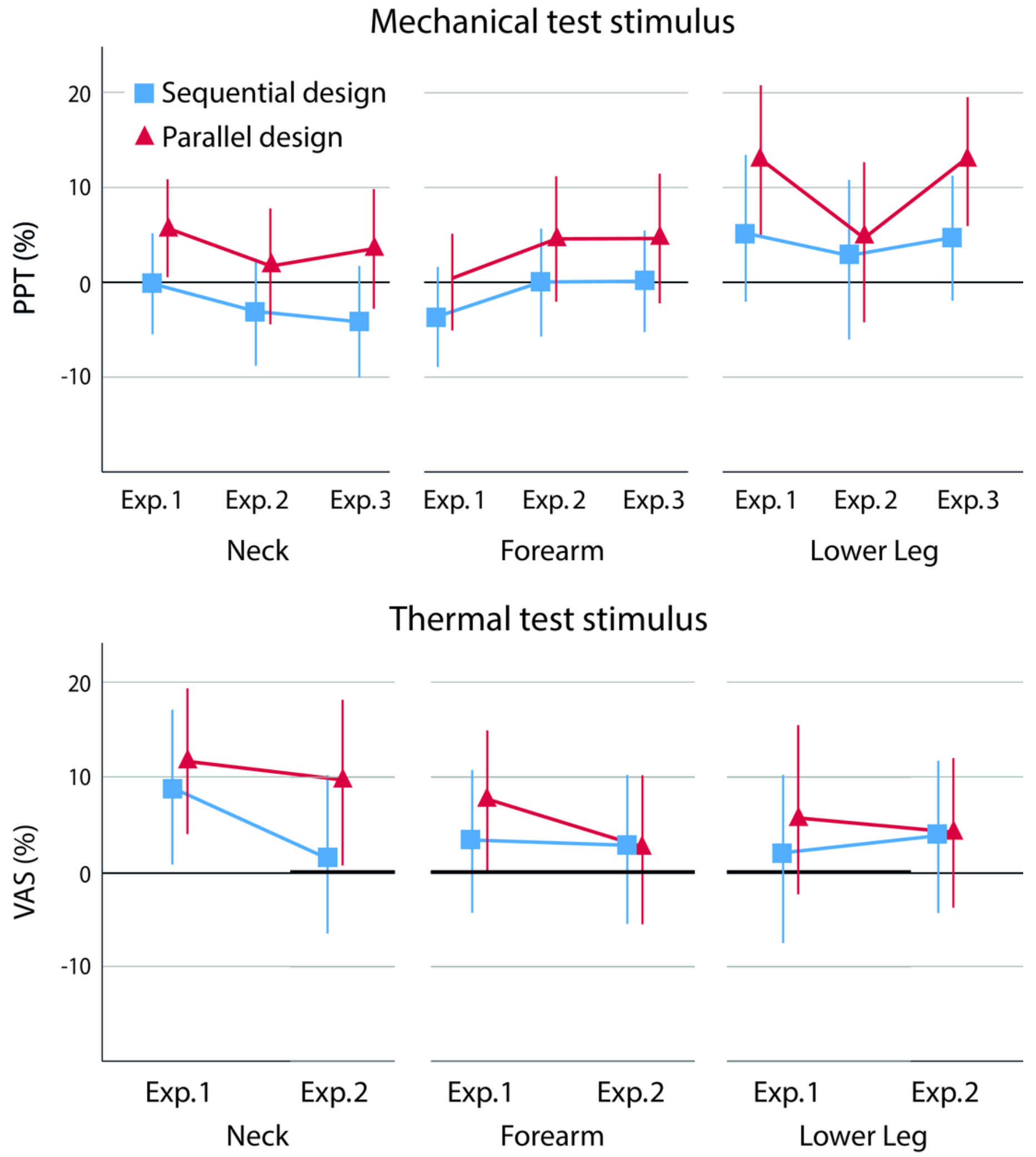




\section{Figure 5}

Quantile regression plots.

Quantile regression plots with estimated regression coefficient for the main effect of design, per $10^{\text {th }}$ quantile, including $95 \%$ confidence intervals and regression estimate based on linear mixed model regression (main analysis), presented per outcome and test location. A negative coefficient indicates that the sequential design has a lower effect compared to the parallel design. 

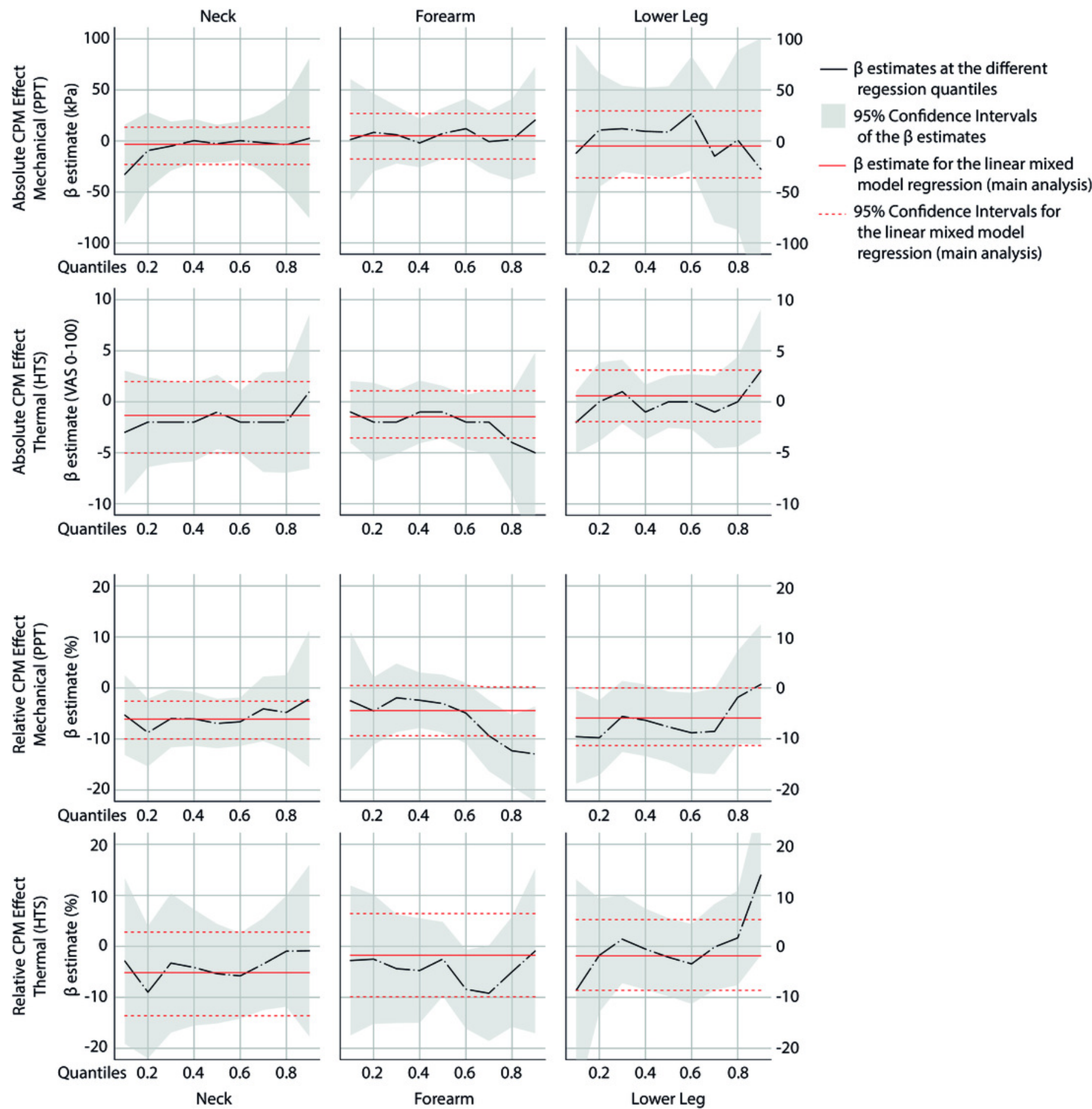